\title{
Effect of surface acidity of cyano-bridged polynuclear metal complexes on catalytic activity for hydrolysis of organophosphates
}

Hiroyasu Tabe, Chihiro Terashima and Yusuke Yamada

\begin{tabular}{|c|l|}
\hline Citation & Catalysis Science \& Technology, 8(18); 4747-4756 \\
\hline Issue Date & 2018-08-09 \\
\hline Type & Journal Article \\
\hline $\begin{array}{c}\text { Textversion } \\
\begin{array}{c}\text { Supplementary } \\
\text { files }\end{array}\end{array}$ & author \\
\hline Supplementary information is available at https://doi.org/10.1039/C8CY01015C. \\
\hline DOI & $\begin{array}{l}\text { The following article has been accepted by Catalysis Science \& Technology. After } \\
\text { it is published, it will be found at https://doi.org/10.1039/C8CY01015C . }\end{array}$ \\
\hline
\end{tabular}

\author{
Self-Archiving by Author(s) \\ Placed on: Osaka City University
}

H. Tabe, C. Terashima and Y. Yamada. (2018). Effect of surface acidity of cyano-bridged polynuclear metal complexes on catalytic activity for hydrolysis of organophosphates. Catalysis Science \& Technology. 8, 4747-4756. DOI: 10.1039/C8CY01015C 


\section{Accepted Manuscript}

This article can be cited before page numbers have been issued, to do this please use: H. Tabe, C.

Terashima and Y. Yamada, Catal. Sci. Technol., 2018, DOI: 10.1039/C8CY01015C.

\section{Catalysis Science \& Technology}
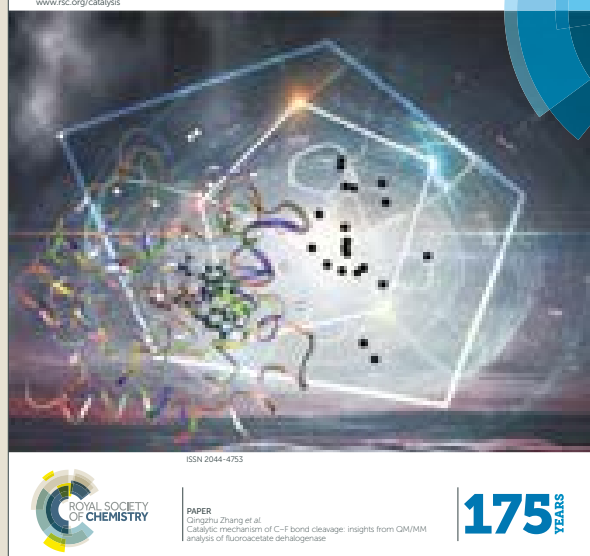

175
This is an Accepted Manuscript, which has been through the Royal Society of Chemistry peer review process and has been accepted for publication.

Accepted Manuscripts are published online shortly after acceptance, before technical editing, formatting and proof reading. Using this free service, authors can make their results available to the community, in citable form, before we publish the edited article. We will replace this Accepted Manuscript with the edited and formatted Advance Article as soon as it is available.

You can find more information about Accepted Manuscripts in the author guidelines.

Please note that technical editing may introduce minor changes to the text and/or graphics, which may alter content. The journal's standard Terms \& Conditions and the ethical guidelines, outlined in our author and reviewer resource centre, still apply. In no event shall the Royal Society of Chemistry be held responsible for any errors or omissions in this Accepted Manuscript or any consequences arising from the use of any information it contains. 
Received 00th January 20xx, Accepted 00th January 20xx

DOI: $10.1039 / x 0 \times x 00000 x$

www.rsc.org/

\title{
Effect of surface acidity of cyano-bridged polynuclear metal complexes on catalytic activity for hydrolysis of organophosphates ${ }^{\dagger}$
}

\author{
Hiroyasu Tabe, Chihiro Terashima and Yusuke Yamada*
}

\begin{abstract}
Heterogeneous catalysis of cyano-bridged polynuclear metal complexes, which were prepared by systematic replacement

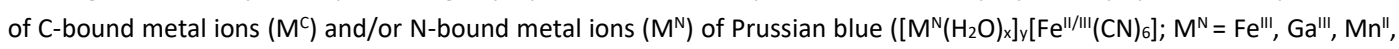

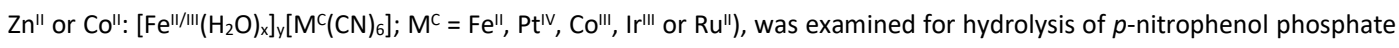
( $p$-NPP) as a model compound of insecticides. Catalytic activity of the complexes was enhanced by employing metal ions in higher oxidation states at $\mathrm{C}$ - and $\mathrm{N}$-bound sites, although only $\mathrm{N}$-bound metal ions act as active sites. The dependence of initial rates for hydrolysis on the initial concentration of $p$-NPP suggested that the rate determining step is the adsorption of $p$-NPP to catalyst surfaces. The surface acidity of each complex estimated by the heat of pyridine desorption is strongly correlated with catalytic activity.
\end{abstract}

\section{Introduction}

Organophosphates constitute the largest class of insecticides and herbicides currently used all over the world. ${ }^{1,2}$ Organophosphates are known as toxic compounds which inhibit acetylcholinesterases to cause a rapidly progressive cholinergic crisis. ${ }^{3}$ Hydrolytic decomposition of organophosphates results in lessening their toxicity. ${ }^{4,5}$ So far, some metalloenzymes and metal oxides have been reported to act as the catalysts for hydrolysis of organophosphates, ${ }^{6-12}$ however, further enhancement of the catalytic activity is demanded, because some organophosphates are highly toxic even at very low concentrations.

Metal complexes have recently emerged as promising candidates as catalysts for hydrolysis of organophosphates because of their high activity and tunability with defined active sites. ${ }^{13-20}$ Recently, coordination polymers composed of metal ions and bridging ligands have attracted much attention, because they act as heterogeneous catalysts, ${ }^{21-49}$ which are beneficial for easy handling, high robustness, high recyclability, etc. The catalytic activity of coordination polymers can be tuned by introducing various functional groups to bridging ligands. ${ }^{48,49}$ The surface acid/base properties of coordination

Department of Applied Chemistry and Bioengineering, Graduate School of Engineering, Osaka City University, Osaka 558-8585, Japan.

E-mail: ymd@a-chem.eng.osaka-cu.ac.jp

$\dagger$ Electronic Supplementary Information (ESI) available: Powder X-ray diffraction patterns (Fig. S1 and S8); infrared spectra (Fig. S2); thermogravimetric analyses (Fig. S3); nitrogen adsorption isotherms (Fig. S4 and S9); time profiles of the formation of catalytic products (Fig. S5-S7); TPD spectra (Fig. S10-S12) elemental analyses by X-ray fluorescence measurements (Table S1); physical parameters of Prussian blue in different morphology (Table S2); and a summary of TPD analyses (Table S3). See DOI: 10.1039/x0xx00000x polymers seem important for hydrolysis of organophosphates, however, the correlation between surface acidity and the catalytic activity of coordination polymers has yet to be clarified. ${ }^{50-56}$

Cyano-bridged polynuclear metal complexes known as Prussian blue analogues are one of the simplest class of coordination polymers, which possess defined active sites with high tunability on the degree of coordinative saturation and electronic structure by choosing an appropriate combination of $\mathrm{C}$ - and N-bound metal ions. A typical formula of the complexes can be expressed as $\left[\mathrm{M}^{\mathrm{N}}\left(\mathrm{H}_{2} \mathrm{O}\right)_{\mathrm{x}}\right]_{\mathrm{y}}\left[\mathrm{M}^{\mathrm{C}}(\mathrm{CN})_{6}\right]$ where the C-bound metal ions and the N-bound metal ions are represented as $\mathrm{M}^{\mathrm{C}}$ and $\mathrm{M}^{\mathrm{N}}$, respectively. The numbers of $\mathrm{x}$ and $\mathrm{y}$ depend on the oxidation states of $\mathrm{M}^{\mathrm{C}}$ and $\mathrm{M}^{\mathrm{N}}$ under consideration of charge neutralisation. In the complexes with $\mathrm{y}>1, \mathrm{M}^{\mathrm{N}}$ ions can act as catalytic active sites, because $\mathrm{M}^{\mathrm{N}}$ ions cannot take an octahedral structure without coordination of extra ligands such as water molecules, which can be liberated from metal ions to provide binding sites for a substrate during the reaction (Fig. 1a). ${ }^{57,58}$ Additionally, replacement of $\mathrm{M}^{\mathrm{C}}$ ions easily modulates the electronic structure of $\mathrm{M}^{\mathrm{N}}$ ions by changing the electron donation ability of a $\mathrm{CN}$ ligand through the $\mathrm{M}^{\mathrm{C}}-\mathrm{CN}$ bond, resulting in enhancement of catalytic activity for redox

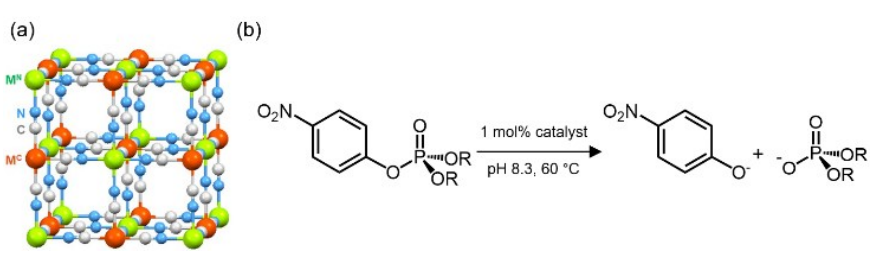

Fig. 1 (a) Partial structure of a cyano-bridged polynuclear metal complex. (b) Scheme of catalytic hydrolysis of organophosphates. $\mathrm{R}=\mathrm{H}$ ( $p$-nitrophenol phosphate, $p$-NPP) or $\mathrm{CH}_{3}$ (paraoxon methyl, $\mathrm{PO}$ ). 
reactions, such as water oxidation, oxygenation of aromatic compounds, etc, ${ }^{59-71}$ while the effect of $\mathrm{M}^{\mathrm{C}}$ ions on catalytic activity of $\mathrm{M}^{\mathrm{N}}$ ions for acid-base reactions has not been scrutinised.

Herein we report catalytic activity of a series of cyanobridged polynuclear metal complexes, $\left[\mathrm{M}^{\mathrm{N}}\left(\mathrm{H}_{2} \mathrm{O}\right)_{\mathrm{x}}\right]_{\mathrm{y}}\left[\mathrm{Fe}^{\mathrm{II} / \mathrm{III}}(\mathrm{CN})_{6}\right]\left(\mathrm{M}^{\mathrm{N}}=\mathrm{Fe}^{\mathrm{III}}, \mathrm{Ga}^{\mathrm{III}}, \mathrm{Mn}^{\mathrm{II}}, \mathrm{Zn}^{\mathrm{II}}\right.$ or $\left.\mathrm{Co}^{\mathrm{II}}\right)$ and $\left[\mathrm{Fe}^{\mathrm{II} / \mathrm{III}}\left(\mathrm{H}_{2} \mathrm{O}\right)_{\mathrm{x}}\right]_{\mathrm{y}}\left[\mathrm{M}^{\mathrm{C}}(\mathrm{CN})_{6}\right]\left(\mathrm{M}^{\mathrm{C}}=\mathrm{Fe}^{\mathrm{II}}, \mathrm{Pt}^{\mathrm{IV}}, \mathrm{Co}^{\mathrm{III}}, \mathrm{Ir}^{\mathrm{III}}\right.$ or $\mathrm{Ru}^{\mathrm{II}}$ ), for hydrolysis of organophosphates (Fig. 1b). Firstly, effect of $\mathrm{M}^{\mathrm{N}}$ ions in $\left[\mathrm{M}^{\mathrm{N}}\left(\mathrm{H}_{2} \mathrm{O}\right)_{\mathrm{x}}\right]_{\mathrm{y}}\left[\mathrm{Fe}^{\mathrm{II} / \mathrm{III}}(\mathrm{CN})_{6}\right]$ on catalysis was examined by employing $\mathrm{Fe}^{\mathrm{III}}, \mathrm{Ga}^{\mathrm{III}}, \mathrm{Mn}^{\mathrm{II}}, \mathrm{Zn}^{\mathrm{II}}$ or $\mathrm{Co}^{\mathrm{II}}$ ions as $\mathrm{M}^{\mathrm{N}}$ ions. Then, effects of $\mathrm{M}^{\mathrm{C}}$ ions on the catalysis of $\mathrm{M}^{\mathrm{N}}=$ $\mathrm{Fe}^{\mathrm{II} / I I I}$ ions were investigated by employing $\mathrm{M}^{\mathrm{C}}$ ions with different valences chosen from $\mathrm{Fe}^{\mathrm{II}}, \mathrm{Pt}^{\mathrm{IV}}, \mathrm{Co}^{\mathrm{III}}, \mathrm{Ir}^{\mathrm{III}}$ and $\mathrm{Ru}^{\mathrm{II}}$. The surface acidity of cyano-bridged polynuclear metal complexes was evaluated by temperature programmed pyridine desorption to correlate with catalytic activity for hydrolysis Finally, the reaction conditions were optimized in terms of $\mathrm{pH}$ of the reaction solution, reaction temperature and morphology of catalytic particles.

\section{Experimental Section}

Materials. All chemicals used for synthesis were obtained from chemical companies and used without further purification. $\mathrm{K}_{3}\left[\mathrm{Co}^{\mathrm{III}}(\mathrm{CN})_{6}\right], \mathrm{K}_{3}\left[\mathrm{Ir}^{\mathrm{III}}(\mathrm{CN})_{6}\right], \mathrm{K}_{4}\left[\mathrm{Pt}^{\mathrm{IV}}(\mathrm{CN})_{6}\right], \mathrm{K}_{2}\left[\mathrm{Ru}^{\mathrm{II}}(\mathrm{CN})_{6}\right]$, paraoxon methyl and 4-(2-hydroxyethyl)-1piperazineethanesulfonic acid (HEPES) were purchased from Sigma-Aldrich Co. LLC. $\mathrm{K}_{3}\left[\mathrm{Fe}^{\mathrm{III}}(\mathrm{CN})_{6}\right], \mathrm{K}_{4}\left[\mathrm{Fe}^{\mathrm{II}}(\mathrm{CN})_{6}\right] \cdot 3 \mathrm{H}_{2} \mathrm{O}$, metal salts, $p$-nitrophenyl phosphate disodium salt hexahydrate and dehydrated pyridine were delivered by Wako Pure Chemical Industries, Ltd. Polyvinylpyrrolidone (K 30, average molecular weight: 40,000) was obtained from Tokyo Chemical Industry Co., Ltd. Purified water was provided by a water purification system, Advantec RFD210TA, where the electronic conductance was $18.2 \mathrm{M} \Omega \mathrm{cm}$. $\left[\mathrm{Fe}^{\mathrm{III}}\left(\mathrm{H}_{2} \mathrm{O}\right)_{1.5}\right]_{4 / 3}\left[\mathrm{Fe}^{\mathrm{II}}(\mathrm{CN})_{6}\right]$ (Prussian blue), $\left[\mathrm{Fe}^{\mathrm{II}}\left(\mathrm{H}_{2} \mathrm{O}\right)_{2}\right]_{1.5}\left[\mathrm{Co}^{\mathrm{III}}(\mathrm{CN})_{6}\right], \mathrm{Fe}^{\mathrm{III}}\left[\mathrm{Co}^{\mathrm{III}}(\mathrm{CN})_{6}\right]$, $\left[\mathrm{Co}^{\mathrm{II}}\left(\mathrm{H}_{2} \mathrm{O}\right)_{2}\right]_{1.5}\left[\mathrm{Fe}^{\mathrm{III}}(\mathrm{CN})_{6}\right]$, $\left[\mathrm{Mn}^{\mathrm{II}}\left(\mathrm{H}_{2} \mathrm{O}\right)_{2}\right]_{1.5}\left[\mathrm{Fe}^{\mathrm{III}}(\mathrm{CN})_{6}\right]$, $\left[\mathrm{Ga}^{\mathrm{III}}\left(\mathrm{H}_{2} \mathrm{O}\right)_{1.5}\right]_{4 / 3}\left[\mathrm{Fe}^{\mathrm{II}}(\mathrm{CN})_{6}\right]$, $\left[\mathrm{Fe}^{\mathrm{II}}\left(\mathrm{H}_{2} \mathrm{O}\right)_{2}\right]_{1.5}\left[\mathrm{Ir}^{\mathrm{III}}(\mathrm{CN})_{6}\right]$ $\left[\mathrm{Zn}^{\mathrm{II}}\left(\mathrm{H}_{2} \mathrm{O}\right)_{2}\right]_{1.5}\left[\mathrm{Fe}^{\mathrm{III}}(\mathrm{CN})_{6}\right]$, $\mathrm{Fe}_{2}\left[\mathrm{Pt}^{\mathrm{IN}}(\mathrm{CN})_{6}\right]$ $\left[\mathrm{Fe}^{\mathrm{II}}\left(\mathrm{H}_{2} \mathrm{O}\right)_{3}\right]_{2}\left[\mathrm{Ru}^{\mathrm{II}}(\mathrm{CN})_{6}\right]$ were synthesised according to literature procedures. ${ }^{70-76}$

Synthesis of $\mathrm{Ga}^{\mathrm{III}}\left[\mathrm{Fe}^{\mathrm{III}}(\mathrm{CN})_{6}\right]$. An aqueous solution of gallium(III) nitrate $(0.18 \mathrm{M}, 2.5 \mathrm{~mL})$ was slowly added to an aqueous solution of potassium ferricyanide $(0.12 \mathrm{M}, 2.5 \mathrm{~mL})$. Then the solution was slowly evaporated to obtain the precipitate. The formed precipitates were collected by filtration and washed with distilled water several times. The precipitates were dried at $60{ }^{\circ} \mathrm{C}$ for $12 \mathrm{~h}$. IR ( $\left.\mathrm{KBr}\right): 3425 \mathrm{~cm}^{-1}\left(v_{\mathrm{OH}}\right), 2121$ $\mathrm{cm}^{-1}\left(v_{\mathrm{CN}}\right), 602 \mathrm{~cm}^{-1}\left(v_{\mathrm{MC}}\right), 498 \mathrm{~cm}^{-1}\left(\delta_{\mathrm{M}-\mathrm{C}-\mathrm{N}}\right)$.

Synthesis of $\mathrm{Fe}^{\mathrm{III}}\left[\mathrm{Ir}^{\mathrm{III}}(\mathrm{CN})_{6}\right]$. An aqueous solution of iron(III) nitrate $(0.12 \mathrm{M}, 2.5 \mathrm{~mL})$ was slowly added to an aqueous solution of potassium hexachloroiridate $(0.12 \mathrm{M}, 2.5$ $\mathrm{mL})$. Then the solution was slowly evaporated to obtain precipitates. The formed precipitates were collected by centrifugation and washed with distilled water several times.
The precipitates were dried at $60{ }^{\circ} \mathrm{C}$ for $12 \mathrm{~h}$. IR $\left(\mathrm{KBBr}_{\mathrm{B}}\right) \mathrm{ie} 3425$ $\mathrm{cm}^{-1}\left(v_{\mathrm{OH}}\right), 2185 \mathrm{~cm}^{-1}\left(v_{\mathrm{CN}}\right)$.

DOI: $10.1039 /$ C8CY01015C

Synthesis of $\left[\mathrm{Fe}^{\mathrm{III}}\left(\mathrm{H}_{2} \mathrm{O}\right)_{1.5}\right]_{4 / 3}\left[\mathrm{Ru}^{\mathrm{II}}(\mathrm{CN})_{6}\right]$. An aqueous solution of iron(III) nitrate $(0.09 \mathrm{M}, 2.5 \mathrm{~mL})$ was slowly added to an aqueous solution of $\mathrm{K}_{2}\left[\mathrm{Ru}^{\mathrm{II}}(\mathrm{CN})_{6}\right](0.12 \mathrm{M}, 2.5 \mathrm{~mL})$ with vigorous stirring for 2 hours. The formed precipitates were collected by filtration and washed with distilled water several times. The precipitates were dried at $60{ }^{\circ} \mathrm{C}$ for $12 \mathrm{~h}$. IR (KBr): $3415 \mathrm{~cm}^{-1}\left(v_{\mathrm{OH}}\right), 2087 \mathrm{~cm}^{-1}\left(v_{\mathrm{CN}}\right), 555 \mathrm{~cm}^{-1}\left(v_{\mathrm{MC}}\right), 484 \mathrm{~cm}^{-1}$ $(\delta \mathrm{M}-\mathrm{C}-\mathrm{N})$.

Shape-controlled synthesis of Prussian blue. Prussian blue in cubic shape (PB-cube) was synthesised by a previously reported procedure with slight modifications. ${ }^{77}$ An aqueous solution of iron(II) sulfate $(12.5 \mathrm{mM}, 60 \mathrm{~mL})$ containing sodium citrate $(42.5 \mathrm{mM})$ was slowly added to an aqueous solution of potassium ferricyanide $(12.5 \mathrm{mM}, 60 \mathrm{~mL})$ with vigorous stirring for $20 \mathrm{~h}$. The formed precipitates were collected by centrifugation and washed with distilled water several times. The precipitates were dried at $60^{\circ} \mathrm{C}$ for $12 \mathrm{~h}$.

Prussian blue in spherical shape (PB-sphere) was prepared according to a previously reported procedure with slight modifications. ${ }^{78}$ An aqueous solution of $\mathrm{K}_{3}\left[\mathrm{Fe}^{\mathrm{III}}(\mathrm{CN})_{6}\right](6.8$ $\mathrm{mM}, 30 \mathrm{~mL})$ containing polyvinylpyrrolidone $(2.5 \mathrm{~g})$ was adjusted to $\mathrm{pH} 1.8$ by adding hydrochloric acid. The mixture was transferred to a Teflon-lined stainless-steel autoclave and heated to $120{ }^{\circ} \mathrm{C}$ for $1 \mathrm{~h}$. The formed precipitates were collected by centrifugation after cooled down to room temperature. The precipitates were washed with distilled water several times and dried at $60{ }^{\circ} \mathrm{C}$ for $12 \mathrm{~h}$.

Physical measurements. UV-vis absorption spectra of reaction solutions were recorded on a JASCO V-770 spectrometer. IR spectra were recorded on a JASCO FT/IR-6200 spectrometer for the samples pelletised with potassium bromide. The atomic ratio of each complex was determined using a Shimadzu EDX-730 X-ray fluorescence spectrometer. Dynamic light scattering (DLS) experiments were conducted at room temperature using a Malvern Instruments Zetasizer Nano S90 Particle Size Analyzer.

$\mathbf{N}_{2}$ adsorption-desorption isotherms. Nitrogen $\left(\mathrm{N}_{2}\right)$ adsorption-desorption isotherms at $-196{ }^{\circ} \mathrm{C}$ were obtained with a Belsorp-mini (BEL Japan, Inc.) within a relative pressure range from 0.01 to $101.3 \mathrm{kPa}$. Weighed samples $(\sim 100 \mathrm{mg})$ were used for adsorption analysis after the removal of physisorbed water at $120{ }^{\circ} \mathrm{C}$ for $1 \mathrm{~h}$ under vacuum conditions. The samples were exposed to $\mathrm{N}_{2}$ and adsorbed amount of $\mathrm{N}_{2}$ was calculated from the change of pressure in a cell after reaching equilibrium at $-196{ }^{\circ} \mathrm{C}$. The total surface area and the outer surface area of each complex were calculated by the Brunauer-Emmett-Teller (BET) method and the $t$-plot method, respectively.

Powder X-ray diffraction. Powder X-ray diffraction patterns were recorded on a Shimadzu XD-3A. Incident X-ray radiation was produced by a Fe X-ray tube operating at $40 \mathrm{kV}$ and $15 \mathrm{~mA}$ with $\mathrm{Fe} K \alpha$ radiation $(\lambda=1.94 \AA)$. The scan rate was $1^{\circ} \mathrm{min}^{-1}$ from $2 \theta=10-80^{\circ}$.

Catalysis evaluation for hydrolysis of organophosphates. A typical procedure for catalysis measurements is as follows: 
HEPES buffer $(100 \mathrm{mM}, \mathrm{pH} 8.3,0.75 \mathrm{~mL})$ containing $p$ nitrophenyl phosphate ( $p$-NPP, $25 \mathrm{mM})$ and a catalyst ([substrate $] /\left[\mathrm{M}^{\mathrm{N}}\right]=100,\left[\mathrm{M}^{\mathrm{N}}\right]$ is an amount of $\mathrm{M}^{\mathrm{N}}$ ions on the active surface sites) was shaken at $900 \mathrm{rpm}$ at $60{ }^{\circ} \mathrm{C}$ and using a heating block mounted on an Eppendorf ThermoMixer C. The reaction vial was removed from the shaker and quickly opened for sampling an aliquot $(10 \mu \mathrm{L})$. The aliquot was diluted with HEPES buffer $(100 \mathrm{mM}$, pH 8.3, 2,490 $\mathrm{LL})$ and analysed by a $\mathrm{UV}-\mathrm{vis}$ spectrophotometer. The conversion of $p$-NPP as a function of reaction time was obtained by monitoring the increase of absorbance of the $p$-nitrophenolate ion $(p-\mathrm{NP})$ at $410 \mathrm{~nm}\left(\varepsilon=1.57 \times 10^{4} \mathrm{M}^{-1} \mathrm{~cm}\right)$. Recycling performance was evaluated by adding the buffer solution containing $p$-NPP to catalysts taken out from the reaction solution.

Temperature-programmed desorption (TPD) of pyridine. TPD measurements using pyridine as a probe molecule were performed by a MicrotracBEL BELCAT II. A typical procedure is as follows: Each complex was pretreated to remove physisorbed water with a flow of helium $(\mathrm{He}, 30 \mathrm{~mL}$ $\min ^{-1}$ ) at $150{ }^{\circ} \mathrm{C}$ for $1 \mathrm{~h}$, and then $5 \mathrm{kPa}$ of pyridine in a $\mathrm{He}$ flow $\left(30 \mathrm{~mL} \mathrm{~min}{ }^{-1}\right)$ was adsorbed at $100{ }^{\circ} \mathrm{C}$ for $30 \mathrm{~min}$. After excess pyridine was removed with a He flow at $150{ }^{\circ} \mathrm{C}$ for 30 min, the temperature of the bed was increased linearly at a rate of $2-10{ }^{\circ} \mathrm{C} \mathrm{min}^{-1}$ from 150 to $550{ }^{\circ} \mathrm{C}$ with a He flow $(30 \mathrm{~mL}$ $\left.\min ^{-1}\right)$. The desorbed pyridine was detected by a thermal conductivity detector (TCD) heated at $135^{\circ} \mathrm{C}$.

\section{Results and discussion}

Effect of N-bound metal ions $\left(\mathrm{M}^{\mathrm{N}}\right)$ of cyano-bridged polynuclear metal complexes $\left(\mathrm{M}^{\mathrm{C}}=\mathrm{Fe}^{\mathrm{II} / \mathrm{III}}\right)$ on catalysis for hydrolysis of organophosphates. A series of cyano-bridged polynuclear metal complexes, $\left[\mathrm{M}^{\mathrm{N}}\left(\mathrm{H}_{2} \mathrm{O}\right)_{\mathrm{x}}\right]_{\mathrm{y}}\left[\mathrm{Fe}^{\mathrm{II} / \mathrm{III}}(\mathrm{CN})_{6}\right]$, including $\left[\mathrm{Fe}^{\mathrm{III}}\left(\mathrm{H}_{2} \mathrm{O}\right)_{1.5}\right]_{4 / 3}\left[\mathrm{Fe}^{\mathrm{II}}(\mathrm{CN})_{6}\right]$ (Prussian blue, $\mathrm{PB}$ ), $\left[\mathrm{Mn}^{\mathrm{II}}\left(\mathrm{H}_{2} \mathrm{O}\right)_{2}\right]_{1.5}\left[\mathrm{Fe}^{\mathrm{III}}(\mathrm{CN})_{6}\right], \quad\left[\mathrm{Co}^{\mathrm{II}}\left(\mathrm{H}_{2} \mathrm{O}\right)_{2}\right]_{1.5}\left[\mathrm{Fe}^{\mathrm{III}}(\mathrm{CN})_{6}\right]$, $\left[\mathrm{Zn}^{\mathrm{II}}\left(\mathrm{H}_{2} \mathrm{O}\right)_{2}\right]_{1.5}\left[\mathrm{Fe}^{\mathrm{III}}(\mathrm{CN})_{6}\right], \quad \mathrm{Ga}^{\mathrm{III}}\left[\mathrm{Fe}^{\mathrm{III}}(\mathrm{CN})_{6}\right] \quad$ and $\left[\mathrm{Ga}^{\mathrm{III}}\left(\mathrm{H}_{2} \mathrm{O}\right)_{1.5}\right]_{4 / 3}\left[\mathrm{Fe}^{\mathrm{II}}(\mathrm{CN})_{6}\right]$, were prepared by mixing an aqueous solution containing metal salts and an aqueous solution of $\mathrm{K}_{3}\left[\mathrm{Fe}^{\mathrm{III}}(\mathrm{CN})_{6}\right]$ or $\mathrm{K}_{4}\left[\mathrm{Fe}^{\mathrm{II}}(\mathrm{CN})_{6}\right]$ to yield precipitates. The compositions of metal ions in the obtained precipitates were determined by X-ray fluorescence spectroscopy (Table 1 and Table S1 in ESI). The molar ratio of $\mathrm{M}^{\mathrm{N}} / \mathrm{M}^{\mathrm{C}}$ of each complex is virtually the same as that predicted from the valence of $\mathrm{M}^{\mathrm{N}}$ and $\mathrm{M}^{\mathrm{C}}$ ions within an experimental error. Some complexes involved small amount of $\mathrm{K}^{+}$ion due to porous structure of cyano-bridged polynuclear metal complexes, however, the contaminated $\mathrm{K}^{+}$ions insignificantly affected on the catalysis as described below.

All the complexes obtained herein provided the same X-ray diffraction (XRD) patterns confirming their cubic structure except for $\left[\mathrm{Zn}^{\mathrm{II}}\left(\mathrm{H}_{2} \mathrm{O}\right)_{2}\right]_{1.5}\left[\mathrm{Fe}^{\mathrm{III}}(\mathrm{CN})_{6}\right]$, which is reported to have several crystal structures (Table 1 and Fig. S1). ${ }^{74}$ The broad peaks for $\left[\mathrm{Fe}^{\mathrm{III}}\left(\mathrm{H}_{2} \mathrm{O}\right)_{1.5}\right]_{4 / 3}\left[\mathrm{Fe}^{\mathrm{II}}(\mathrm{CN})_{6}\right]$ resulted from poor crystallinity or small-sized crystallites (Fig. S1a). The CN stretching band $\left(v_{\mathrm{CN}}\right)$ of $\left[\mathrm{M}^{\mathrm{N}}\left(\mathrm{H}_{2} \mathrm{O}\right)_{\mathrm{x}}\right]_{\mathrm{y}}\left[\mathrm{Fe}^{\mathrm{II} / \mathrm{III}}(\mathrm{CN})_{6}\right]$ complexes observed by infrared (IR) spectroscopy shifted to the higher wavenumber side compared with those of eachwprecursor because $\mathrm{CN}^{-}$acts as a $\sigma$ donor to decreaseothe eleetronf dernsity on the antibonding $\sigma^{*}$ orbital by coordinating to metal ions (Table 1 and Fig. S2). ${ }^{79}$ The numbers of uncoordinated water molecules contained in $\left[\mathrm{M}^{\mathrm{N}}\left(\mathrm{H}_{2} \mathrm{O}\right)_{\mathrm{x}}\right]_{y}\left[\mathrm{Fe}^{\mathrm{II} / \mathrm{III}}(\mathrm{CN})_{6}\right]$ were calculated by the weight losses in thermogravimetric analyses (Fig. S3).

$\mathrm{N}_{2}$ adsorption-desorption isotherms of the complexes were obtained at $-196{ }^{\circ} \mathrm{C}$ to investigate surface areas and pore structures (Fig. S4). The total surface areas were determined by the Brunauer-Emmett-Teller (BET) method as well as the external surface areas by the $t$-plot method (Table 1). The type I isotherms observed for all the complexes except $\left[\mathrm{Fe}^{\mathrm{III}}\left(\mathrm{H}_{2} \mathrm{O}\right)_{1.5}\right]_{4 / 3}\left[\mathrm{Fe}^{\mathrm{II}}(\mathrm{CN})_{6}\right]$ suggest a homogeneous distribution of micropores ( $c a .0 .6 \mathrm{~nm}$ determined by the microporous (MP) method) formed by the cubic lattice structure. Analysis of the isotherms with the Barrett-Joyner-Halenda (BJH) method confirmed the absence of meso- or macropores, suggesting that the catalytic reactions of organophosphates $(c a .10 \AA)$ proceed mainly on the external surfaces of complexes. The type IV isotherm characteristic for mesoporous materials was obtained for $\left[\mathrm{Fe}^{\mathrm{III}}\left(\mathrm{H}_{2} \mathrm{O}\right)_{1.5}\right]_{4 / 3}\left[\mathrm{Fe}^{\mathrm{II}}(\mathrm{CN})_{6}\right]$ (Fig. S4a). The size of mesopores of $\left[\mathrm{Fe}^{\mathrm{III}}\left(\mathrm{H}_{2} \mathrm{O}\right)_{1.5}\right]_{4 / 3}\left[\mathrm{Fe}^{\mathrm{II}}(\mathrm{CN})_{6}\right]$ determined by the $\mathrm{BJH}$ method was around $3.5 \mathrm{~nm}$. The powder XRD of $\left[\mathrm{Fe}^{\mathrm{III}}\left(\mathrm{H}_{2} \mathrm{O}\right)_{1.5}\right]_{4 / 3}\left[\mathrm{Fe}^{\mathrm{II}}(\mathrm{CN})_{6}\right]$ clearly indicated that $\left[\mathrm{Fe}^{\mathrm{III}}\left(\mathrm{H}_{2} \mathrm{O}\right)_{1.5}\right]_{4 / 3}\left[\mathrm{Fe}^{\mathrm{II}}(\mathrm{CN})_{6}\right]$ is isostructural to other complexes although the particles sizes are smaller, thus, the Type IV isothermal behaviour resulted from accidentally formed homogeneous gaps among $\left[\mathrm{Fe}^{\mathrm{III}}\left(\mathrm{H}_{2} \mathrm{O}\right)_{1.5}\right]_{4 / 3}\left[\mathrm{Fe}^{\mathrm{II}}(\mathrm{CN})_{6}\right]$ particles

Hydrolysis of $p$-nitrophenyl phosphate $(p$-NPP) and paraoxon methyl (PO) was examined at $60{ }^{\circ} \mathrm{C}$ in HEPES buffer $(100 \quad \mathrm{mM}, \quad \mathrm{pH} \quad 8.3, \quad 0.75 \quad \mathrm{~mL})$ containing $\left[\mathrm{M}^{\mathrm{N}}\left(\mathrm{H}_{2} \mathrm{O}\right)_{\mathrm{x}}\right]_{\mathrm{y}}\left[\mathrm{Fe}^{\mathrm{II} / \mathrm{III}}(\mathrm{CN})_{6}\right]\left([\right.$ substrate $] /\left[\mathrm{M}^{\mathrm{N}}\right]=100 ;\left[\mathrm{M}^{\mathrm{N}}\right]$ is the amount of $\mathrm{M}^{\mathrm{N}}$ ions on the external surface of $\left.\left[\mathrm{M}^{\mathrm{N}}\left(\mathrm{H}_{2} \mathrm{O}\right)_{\mathrm{x}}\right]_{\mathrm{y}}\left[\mathrm{Fe}^{\mathrm{II} / \mathrm{III}}(\mathrm{CN})_{6}\right]\right)$. The amount of the resulting $p$ nitrophenolate ion $\left(\lambda_{\max }=410 \mathrm{~nm}\right)$ was determined by UV-vis spectroscopy (Fig. 2a). ${ }^{43}$ All of the $\left[\mathrm{M}^{\mathrm{N}}\left(\mathrm{H}_{2} \mathrm{O}\right)_{\mathrm{x}}\right]_{\mathrm{y}}\left[\mathrm{Fe}^{\mathrm{II} / \mathrm{III}}(\mathrm{CN})_{6}\right]$ catalysed hydrolysis of $p$-NPP and PO (Table 2, Fig. S5 and $\mathrm{S} 6)$. On the other hand, no catalytic activity observed for $\mathrm{Fe}^{3+}$ ions, which might be leached from $\left[\mathrm{M}^{\mathrm{N}}\left(\mathrm{H}_{2} \mathrm{O}\right)_{\mathrm{x}}\right]_{\mathrm{y}}\left[\mathrm{Fe}^{\mathrm{II} / \mathrm{III}}(\mathrm{CN})_{6}\right]$,

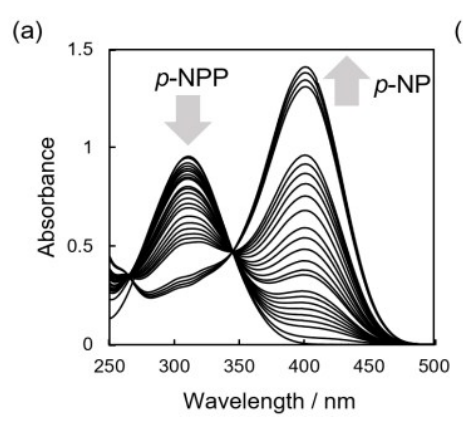

(b)

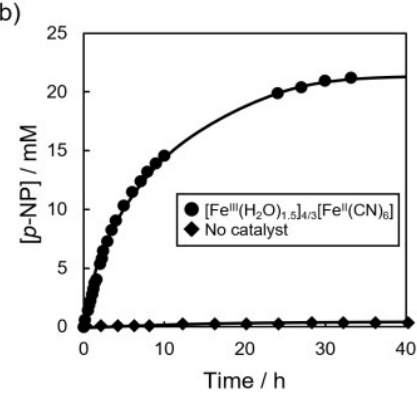

Fig. 2 (a) UV-Vis spectral change by hydrolysis of $p$-nitrophenyl phosphate ( $p$-NPP $25 \mathrm{mM})$ in a HEPES buffer (100 mM, pH 8.3, $0.75 \mathrm{~mL})$ containing $\left[\mathrm{Fe}^{\prime \prime \prime}\left(\mathrm{H}_{2} \mathrm{O}\right)_{1.5}\right]_{4 / 3}\left[\mathrm{Fe}^{\prime \prime}(\mathrm{CN})_{6}\right](6.6 \mathrm{mg}$, [Substrate]/[Fe] $=100)$ at $60{ }^{\circ} \mathrm{C}$. The peak at 410 $\mathrm{nm}$ assignable to $p$-nitrophenol ( $p$-NP) grew, accompanied with decay of the peak at $315 \mathrm{~nm}$ assignable to $p$-NPP. (b) Time profiles of the $p$-NP formation in the presence and absence of $\left[\mathrm{Fe}^{\prime \prime \prime}\left(\mathrm{H}_{2} \mathrm{O}\right)_{1.5}\right]_{4 / 3}\left[\mathrm{Fe}^{\prime \prime}(\mathrm{CN})_{6}\right]$. 


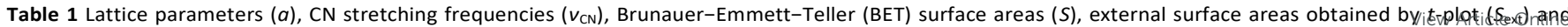

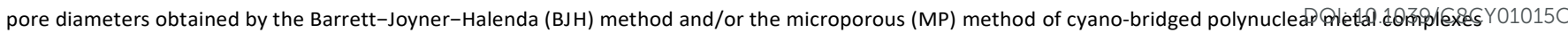

\begin{tabular}{|c|c|c|c|c|c|}
\hline Complex & $a / \AA^{a}$ & $v_{\mathrm{CN}} / \mathrm{cm}^{-1 a}$ & $S / \mathrm{m}^{2} \mathrm{~g}^{-1 a}$ & $S_{\text {ext }} / \mathrm{m}^{2} \mathrm{~g}^{-1 a}$ & Pore diameter / $\mathrm{nm}$ \\
\hline$\left[\mathrm{Fe}^{\mathrm{III}}\left(\mathrm{H}_{2} \mathrm{O}\right)_{1.5}\right]_{4 / 3}\left[\mathrm{Fe}^{\mathrm{II}}(\mathrm{CN})_{6}\right]^{\mathrm{b}}$ & 10.2 & 2080 & 384 & 9 & 3.5 \\
\hline$\left[\mathrm{Mn}^{\mathrm{II}}\left(\mathrm{H}_{2} \mathrm{O}\right)_{2}\right]_{1.5}\left[\mathrm{Fe}^{\mathrm{III}}(\mathrm{CN})_{6}\right]$ & 10.5 & 2149 & 933 & 5 & 0.6 \\
\hline$\left[\mathrm{Co}^{\mathrm{II}}\left(\mathrm{H}_{2} \mathrm{O}\right)_{2}\right]_{1.5}\left[\mathrm{Fe}^{\mathrm{III}}(\mathrm{CN})_{6}\right]$ & 10.3 & 2168 & 685 & 51 & 0.7 \\
\hline$\left[\mathrm{Zn}^{\mathrm{II}}\left(\mathrm{H}_{2} \mathrm{O}\right)_{2}\right]_{1.5}\left[\mathrm{Fe}^{\mathrm{III}}(\mathrm{CN})_{6}\right]$ & 10.4 & 2160 & 836 & 12 & 0.6 \\
\hline $\mathrm{Ga}^{\mathrm{III}}\left[\mathrm{Fe}^{\mathrm{III}}(\mathrm{CN})_{6}\right]$ & 10.2 & 2121 & 202 & 7 & 0.7 \\
\hline$\left[\mathrm{Ga}^{\mathrm{III}}\left(\mathrm{H}_{2} \mathrm{O}\right)_{1.5}\right]_{4 / 3}\left[\mathrm{Fe}^{\mathrm{II}}(\mathrm{CN})_{6}\right]$ & 10.1 & 2119 & 361 & 9 & 0.6 \\
\hline $\mathrm{Fe}^{\mathrm{II}}\left[\mathrm{Pt}^{\mathrm{IV}}(\mathrm{CN})_{6}\right]$ & 10.6 & 2229 & 5 & 5 & 0.7 \\
\hline $\mathrm{Fe}^{\mathrm{III}}\left[\mathrm{Co}^{\mathrm{III}}(\mathrm{CN})_{6}\right]$ & 10.2 & 2185 & 72 & 5 & 0.7 \\
\hline $\mathrm{Fe}^{\mathrm{III}}\left[\operatorname{Ir}^{\mathrm{IIII}}(\mathrm{CN})_{6}\right]$ & 10.5 & 2185 & 136 & 5 & 0.7 \\
\hline$\left[\mathrm{Fe}^{\mathrm{III}}\left(\mathrm{H}_{2} \mathrm{O}\right)_{1.5}\right]_{4 / 3}\left[\mathrm{Ru}^{\mathrm{II}}(\mathrm{CN})_{6}\right]$ & 10.5 & 2087 & 213 & 18 & 0.7 \\
\hline$\left[\mathrm{Fe}^{\mathrm{II}}\left(\mathrm{H}_{2} \mathrm{O}\right)_{2}\right]_{1.5}\left[\mathrm{Co}^{\mathrm{III}}(\mathrm{CN})_{6}\right]$ & 10.3 & 2166 & 172 & 27 & 0.7 \\
\hline$\left[\mathrm{Fe}^{\mathrm{II}}\left(\mathrm{H}_{2} \mathrm{O}\right)_{2}\right]_{1.5}\left[\operatorname{II}^{\mathrm{III}}(\mathrm{CN})_{6}\right]$ & 10.5 & 2181 & 29 & 11 & 0.7 \\
\hline$\left[\mathrm{Fe}^{\mathrm{II}}\left(\mathrm{H}_{2} \mathrm{O}\right)_{3}\right]_{2}\left[\mathrm{Ru}^{\mathrm{II}}(\mathrm{CN})_{6}\right]$ & 10.5 & 2098 & 26 & 23 & 0.7 \\
\hline
\end{tabular}

${ }^{a}$ X-ray diffraction patterns, IR spectra, $\mathrm{N}_{2}$ adsorption-desorption isotherms are shown in the Supporting Information, Fig. S1, S2 and S4, respectively. ${ }^{b}$ The Fe ions in $\left[\mathrm{Fe}^{\mathrm{III}}\left(\mathrm{H}_{2} \mathrm{O}\right)_{1.5}\right]_{4 / 3}\left[\mathrm{Fe}^{\mathrm{II}}(\mathrm{CN})_{6}\right]$ are in mixed valence states.

Table 2 Comparison of catalytic activity of various $\left[\mathrm{M}^{\mathrm{N}}\left(\mathrm{H}_{2} \mathrm{O}\right)_{x}\right]_{y}\left[\mathrm{M}^{\mathrm{C}}(\mathrm{CN})_{6}\right]$ for hydrolysis of organophosphates

\begin{tabular}{|c|c|c|c|c|c|}
\hline Catalyst & Substrate & $\begin{array}{l}\text { Catalyst loaded / } \\
\mathrm{mg}^{a}\end{array}$ & Conversion $(\%)^{b}$ & $\mathrm{TOF} / \mathrm{h}^{-1 b}$ & $v_{0} / \mathrm{mol} \mathrm{L}^{-1} \mathrm{~h}^{-1 c}$ \\
\hline$\left[\mathrm{Fe}^{\mathrm{IIII}}\left(\mathrm{H}_{2} \mathrm{O}\right)_{1.5}\right]_{4 / 3}\left[\mathrm{Fe}^{\mathrm{II}}(\mathrm{CN})_{6}\right](\mathrm{PB})$ & $p$-NPP & 6.7 & 79 & 3.3 & $3.1 \times 10^{-3}$ \\
\hline$\left[\mathrm{Mn}^{\mathrm{II}}\left(\mathrm{H}_{2} \mathrm{O}\right)_{2}\right]_{1.5}\left[\mathrm{Fe}^{\mathrm{III}}(\mathrm{CN})_{6}\right]$ & $p$-NPP & 10.8 & 46 & 1.9 & $6.5 \times 10^{-3}$ \\
\hline$\left[\mathrm{Co}^{\mathrm{II}}\left(\mathrm{H}_{2} \mathrm{O}\right)_{2}\right]_{1.5}\left[\mathrm{Fe}^{\mathrm{III}}(\mathrm{CN})_{6}\right]$ & $p$-NPP & 1.0 & 4 & 0.17 & $3.2 \times 10^{-5}$ \\
\hline$\left[\mathrm{Zn}^{\mathrm{II}}\left(\mathrm{H}_{2} \mathrm{O}\right)_{2}\right]_{1.5}\left[\mathrm{Fe}^{\mathrm{III}}(\mathrm{CN})_{6}\right]$ & $p$-NPP & 3.7 & 5 & 0.21 & $1.2 \times 10^{-4}$ \\
\hline $\mathrm{Ga}^{\mathrm{III}}\left[\mathrm{Fe}^{\mathrm{III}}(\mathrm{CN})_{6}\right]$ & $p$-NPP & 8.2 & 15 & 0.63 & $6.1 \times 10^{-3}$ \\
\hline$\left[\mathrm{Ga}^{\mathrm{III}}\left(\mathrm{H}_{2} \mathrm{O}\right)_{1.5}\right]_{4 / 3}\left[\mathrm{Fe}^{\mathrm{II}}(\mathrm{CN})_{6}\right]$ & $p$-NPP & 5.7 & 26 & 0.13 & $1.1 \times 10^{-4}$ \\
\hline $\mathrm{Fe}^{\mathrm{III}}\left[\mathrm{Co}^{\mathrm{III}}(\mathrm{CN})_{6}\right]$ & $p$-NPP & 12.1 & 95 & 4.0 & $5.3 \times 10^{-3}$ \\
\hline $\mathrm{Fe}^{\mathrm{III}}\left[\mathrm{Ir}^{\mathrm{III}}(\mathrm{CN})_{6}\right]$ & $p$-NPP & 18.8 & 90 & 3.8 & $4.9 \times 10^{-3}$ \\
\hline$\left[\mathrm{Fe}^{\mathrm{III}}\left(\mathrm{H}_{2} \mathrm{O}\right)_{1.5}\right]_{4 / 3}\left[\mathrm{Ru}^{\mathrm{II}}(\mathrm{CN})_{6}\right]$ & $p$-NPP & 3.4 & 81 & 3.4 & $4.1 \times 10^{-3}$ \\
\hline$\left[\mathrm{Fe}^{\mathrm{II}}\left(\mathrm{H}_{2} \mathrm{O}\right)_{2}\right]_{1.5}\left[\mathrm{Co}^{\mathrm{III}}(\mathrm{CN})_{6}\right]$ & $p$-NPP & 1.8 & 27 & 1.1 & $1.0 \times 10^{-3}$ \\
\hline$\left[\mathrm{Fe}^{\mathrm{III}}\left(\mathrm{H}_{2} \mathrm{O}\right)_{2}\right]_{1.5}\left[\operatorname{Ir}^{\mathrm{III}}(\mathrm{CN})_{6}\right]$ & $p$-NPP & 4.7 & 50 & 2.1 & $2.2 \times 10^{-3}$ \\
\hline$\left[\mathrm{Fe}^{\mathrm{II}}\left(\mathrm{H}_{2} \mathrm{O}\right)_{3}\right]_{2}\left[\mathrm{Ru}^{\mathrm{II}}(\mathrm{CN})_{6}\right]$ & $p$-NPP & 2.0 & 31 & 1.3 & $5.1 \times 10^{-4}$ \\
\hline $\mathrm{Fe}^{\mathrm{II}}\left[\mathrm{Pt}^{\mathrm{IV}}(\mathrm{CN})_{6}\right]$ & $p$-NPP & 10.1 & 38 & 1.6 & $2.5 \times 10^{-3}$ \\
\hline No catalyst & $p$-NPP & - & 3 & - & $4.7 \times 10^{-6}$ \\
\hline$\left[\mathrm{Fe}^{\mathrm{III}}\left(\mathrm{H}_{2} \mathrm{O}\right)_{1.5}\right]_{4 / 3}\left[\mathrm{Fe}^{\mathrm{II}}(\mathrm{CN})_{6}\right]$ & $\mathrm{PO}$ & 6.7 & 5 & 0.21 & $1.6 \times 10^{-3}$ \\
\hline $\mathrm{Fe}^{\mathrm{III}}\left[\mathrm{Co}^{\mathrm{III}}(\mathrm{CN})_{6}\right]$ & $\mathrm{PO}$ & 1.8 & 2 & 0.083 & $2.4 \times 10^{-4}$ \\
\hline No catalyst & PO & - & 1 & - & $1.7 \times 10^{-4}$ \\
\hline
\end{tabular}

${ }^{a}[$ substrate $] /\left[\mathrm{M}^{\mathrm{N}}\right]=100 .\left[\mathrm{M}^{\mathrm{N}}\right]$ is calculated from the number of $\mathrm{M}^{\mathrm{N}}$ atoms on the outer surface of complexes dispersed in reaction solutions. ${ }^{b}$ Conversions and turn-over frequencies (TOF) were calculated using the yield of $p$-nitrophenol $\left(p\right.$-NP) in $24 \mathrm{~h}$. ${ }^{c}$ Initial reaction rates $\left(v_{0}\right)$ were measured in the initial 10 $\min$.

evidenced by reactions performed in the presence of $\mathrm{Fe}^{3+}$ ions (Fig. S7) suggests that $\left[\mathrm{M}^{\mathrm{N}}\left(\mathrm{H}_{2} \mathrm{O}\right)_{\mathrm{x}}\right]_{\mathrm{y}}\left[\mathrm{Fe}^{\mathrm{II} / \mathrm{III}}(\mathrm{CN})_{6}\right]$ act as heterogeneous catalysts. The highest $p$-NPP conversion of $79 \%$ $(24 \mathrm{~h})$ with the initial reaction rate $\left(v_{0}\right)$ of $3.1 \times 10^{-3} \mathrm{~mol} \mathrm{~L}^{-1} \mathrm{~h}^{-1}$ $(<1 \mathrm{~h})$ was obtained for the reaction system employing $\left[\mathrm{Fe}^{\mathrm{III}}\left(\mathrm{H}_{2} \mathrm{O}\right)_{1.5}\right]_{4 / 3}\left[\mathrm{Fe}^{\mathrm{II}}(\mathrm{CN})_{6}\right]$ as the catalyst (Fig. 2b). The oxidation state of $\mathrm{Fe}$ ions in the $\mathrm{M}^{\mathrm{N}}$ position is in between +2 and +3 , because $\mathrm{Fe}$ ions in $\left[\mathrm{Fe}^{\mathrm{III}}\left(\mathrm{H}_{2} \mathrm{O}\right)_{1.5}\right]_{4 / 3}\left[\mathrm{Fe}^{\mathrm{II}}(\mathrm{CN})_{6}\right]$ take mixed valence states. Moreover, $v_{0}$ for the reaction system using $\mathrm{Ga}^{\mathrm{III}}\left[\mathrm{Fe}^{\mathrm{III}}(\mathrm{CN})_{6}\right]$ is almost 50 times higher than that using $\left[\mathrm{Ga}^{\mathrm{III}}\left(\mathrm{H}_{2} \mathrm{O}\right)_{1.5}\right]_{4 / 3}\left[\mathrm{Fe}^{\mathrm{II}}(\mathrm{CN})_{6}\right]$, which contains $\mathrm{Fe}$ ions in the lower oxidation state at $\mathrm{M}^{\mathrm{C}}$ sites. These results suggest that both $\mathrm{M}^{\mathrm{N}}$ and $\mathrm{M}^{\mathrm{C}}$ ions in higher oxidation states enhance catalysis.
Effect of C-bound metal ions $\left(\mathrm{M}^{\mathrm{C}}\right)$ of cyano-bridged polynuclear metal complexes $\left(\mathrm{M}^{\mathrm{N}}=\mathrm{Fe}^{\mathrm{II} / \mathrm{III}}\right)$ for hydrolysis of organophosphates. A series of cyano-bridged polynuclear metal complexes having N-bound $\mathrm{Fe}$ ions, $\left(\left[\mathrm{Fe}^{\mathrm{II} / \mathrm{III}}\left(\mathrm{H}_{2} \mathrm{O}\right)_{\mathrm{x}}\right]_{y}\left[\mathrm{M}^{\mathrm{C}}(\mathrm{CN})_{6}\right]\left(\mathrm{M}^{\mathrm{C}}=\mathrm{Fe}^{\mathrm{III}}, \mathrm{Pt}^{\mathrm{IV}}, \mathrm{Co}^{\mathrm{III}}, \mathrm{Ir}^{\mathrm{III}}\right.\right.$ or $\left.\mathrm{Ru}^{\mathrm{II}}\right)$, were prepared by mixing an aqueous solution containing potassium hexacyanometallate and $\mathrm{Fe}$ ion sources, $\mathrm{FeSO}_{4}$ or $\mathrm{Fe}\left(\mathrm{NO}_{3}\right)_{3}$ (Table 1 and Fig. S1-S4). The complexes provided XRD patterns indicating their cubic structure. Broader diffraction peaks of $\mathrm{Fe}^{\mathrm{III}}\left[\mathrm{Co}^{\mathrm{III}}(\mathrm{CN})_{6}\right]$ evidenced poor crystallinity or small-sized crystallites as same as $\left[\mathrm{Fe}^{\mathrm{III}}\left(\mathrm{H}_{2} \mathrm{O}\right)_{1.5}\right]_{4 / 3}\left[\mathrm{Fe}^{\mathrm{II}}(\mathrm{CN})_{6}\right]$ (Fig. S1g).

A series of $\left[\mathrm{Fe}^{\mathrm{II} / \mathrm{II}}\left(\mathrm{H}_{2} \mathrm{O}\right)_{\mathrm{x}}\right]_{y}\left[\mathrm{M}^{\mathrm{C}}(\mathrm{CN})_{6}\right]$ complexes were examined as catalysts for hydrolysis of $p$-NPP in HEPES buffer (100 mM, pH 8.3) (Table 2 and Fig. S5). The highest 
conversion of $95 \%(24 \mathrm{~h})$ with the highest $v_{0}$ of $5.3 \times 10^{-3} \mathrm{~mol}$ $\mathrm{L}^{-1} \mathrm{~h}^{-1}$ was achieved for the reaction system using $\mathrm{Fe}^{\mathrm{III}}\left[\mathrm{Co}^{\mathrm{III}}(\mathrm{CN})_{6}\right]$. The catalytic conversion, TON and $v_{0}$ for the reaction systems using $\left[\mathrm{Fe}^{\mathrm{III}}\left(\mathrm{H}_{2} \mathrm{O}\right)_{\mathrm{x}}\right]_{\mathrm{y}}\left[\mathrm{M}^{\mathrm{C}}(\mathrm{CN})_{6}\right]\left(\mathrm{M}^{\mathrm{C}}=\mathrm{Co}^{\mathrm{III}}\right.$, $\mathrm{Ir}^{\mathrm{III}}$ and $\left.\mathrm{Ru}^{\mathrm{II}}\right)$ containing trivalent $\mathrm{Fe}$ ions were higher than those using $\left[\mathrm{Fe}^{\mathrm{II}}\left(\mathrm{H}_{2} \mathrm{O}\right)_{\mathrm{x}}\right]_{\mathrm{y}}\left[\mathrm{M}^{\mathrm{C}}(\mathrm{CN})_{6}\right]$ containing divalent $\mathrm{Fe}$ ions. The complexes possessing $\mathrm{M}^{\mathrm{C}}$ in high oxidation states such as $\mathrm{Pt}^{\mathrm{IV}}, \mathrm{Ir}^{\mathrm{III}}$ and $\mathrm{Co}^{\mathrm{III}}$ typically provide increased conversion and $k$ compared with complexes having $\mathrm{Ru}^{\mathrm{II}}$, which is in the lowest oxidation state. Thus, the presence of $\mathrm{M}^{\mathrm{C}}$ and/or $\mathrm{M}^{\mathrm{N}}$ in higher oxidation states in $\left[\mathrm{Fe}^{\mathrm{II} / I I}\left(\mathrm{H}_{2} \mathrm{O}\right)_{\mathrm{x}}\right]_{y}\left[\mathrm{M}^{\mathrm{C}}(\mathrm{CN})_{6}\right]$ is important to achieve high catalytic activity.

Rate determining step for hydrolysis of organophosphates. The rate-determining step in the catalytic hydrolysis was determined by examination of the initial reaction rates dependent on the concentration of the substrate. Based on the Langmuir-Hinshelwood's kinetic model, the reaction occurs through five reaction steps (R1-R5): the specific adsorption of $p$-NPP and $\mathrm{H}_{2} \mathrm{O}$ onto unoccupied surface

$$
\begin{aligned}
& \mathrm{H}_{2} \mathrm{O}+\mathrm{Cat} \rightleftarrows \mathrm{H}_{2} \mathrm{O} \cdot \mathrm{Cat} \\
& p \text {-NPP }+ \text { Cat } \rightleftarrows p \text {-NPP. Cat } \\
& p \text {-NPP.Cat }+\mathrm{H}_{2} \mathrm{O} \cdot \mathrm{Cat} \rightleftarrows p \text {-NP.Cat }+ \text { Phos } \cdot \mathrm{Cat} \\
& p \text {-NP. Cat } \rightleftarrows p \text {-NP }+ \text { Cat } \\
& \mathrm{Phos} \cdot \mathrm{Cat} \rightleftarrows \mathrm{Phos}+\mathrm{Cat}
\end{aligned}
$$

sites of catalysts (Cat) yielding surface complexes $\left(\mathrm{H}_{2} \mathrm{O} \cdot \mathrm{Cat}\right.$ and $p$-NPP.Cat), the reaction to form the surface complexes of $p$-NP and phosphate ion ( $p$-NP.Cat and Phos - Cat, respectively), and the desorption of products to obtain free $p$-NP and phosphate ions (Phos). Herein, the respective rate constants for Rns $(\mathrm{n}=1-5)$ are $k_{\mathrm{n}}$ and $k_{-\mathrm{n}}$ where $K_{\mathrm{n}}$ is defined as $K_{\mathrm{n}}=k_{\mathrm{n}} / k_{-\mathrm{n}}$. The concentration of $\mathrm{H}_{2} \mathrm{O}$ remains relatively constant, because $\mathrm{H}_{2} \mathrm{O}$ is supplied in large excess. Thus, the equilibrium constant $K_{1}{ }^{\prime}$ is defined as $K_{1}{ }^{\prime}=K_{1}\left[\mathrm{H}_{2} \mathrm{O}\right]$. A surface coverage by compound $\mathrm{X}$ is now $\theta \mathrm{x}$, where $\theta_{\text {total }}$ is the fraction of occupied sites, thus $\theta_{\text {total }}=\theta_{\mathrm{H} 2 \mathrm{O}}+\theta_{p \text {-NPP }}+\theta_{p \text {-NP }}+\theta_{\text {Phos. }}$ The following two mechanisms can be proposed.

Mechanism 1. When the surface reaction (R3) occurs more quickly than the formation of surface complex with substrates (R2), the overall rate of catalytic hydrolysis should depend on the rate of R2. The adsorption/desorption equilibrium is written by eqs. $1-4$. The overall rate equation for catalytic hydrolysis

$$
\begin{aligned}
& k_{1}\left[\mathrm{H}_{2} \mathrm{O}\right]\left(1-\theta_{\text {total }}\right)=k_{-1} \theta_{\mathrm{H} 2 \mathrm{O}} \\
& k_{3} \theta_{\mathrm{H}_{2} \mathrm{O}} \theta_{p-\mathrm{NPP}}=k_{-3} \theta_{p-\mathrm{NP}} \theta_{\mathrm{Phos}} \\
& k_{4} \theta_{p-\mathrm{NP}}=k-4[p-\mathrm{NP}]\left(1-\theta_{\text {total }}\right) \\
& k_{-5} \theta_{\mathrm{Phos}}=k_{5}[\mathrm{Phos}]\left(1-\theta_{\text {total }}\right)
\end{aligned}
$$

described as $v$ in eq. 5 . The back reactions can be ignored at the

$$
v=k_{2}[p-\mathrm{NPP}]\left(1-\theta_{\text {total }}\right)-k_{-2} \theta_{p-\mathrm{NPP}}
$$$$
K_{4} K_{5}\left(k_{2} K_{1} K_{3}[p-\mathrm{NPP}]-k_{-2} K_{4} K_{5}[p-\mathrm{NP}][\mathrm{Phos}]\right)
$$

$=\overline{K_{1} K_{3} K_{4} K_{5}+K_{3} K_{4} K_{5}+[p-\mathrm{NP}][\mathrm{Phos}]+K_{1^{\prime}} K_{3} K_{5}[p-\mathrm{NP}]+K_{1} K_{3} K_{4}[\mathrm{Phos}]}$

beginning of catalytic hydrolysis $([p-\mathrm{NP}] \simeq 0,[\mathrm{Phos}] \simeq 0)$ to obtain the initial reaction rate $\left(v_{0}\right)$ described by a linear function, eq. 6 , using the initial concentration of $p$-NPP $\left([p-\mathrm{NPP}]_{0}\right)$.

$$
v_{0}=\frac{k_{2} K_{1}^{\prime}[p-\mathrm{NPP}]_{0}}{K_{1}^{\prime}+1}
$$

Mechanism 2. In this mechanism, the reaction of substrates adsorbed on the catalyst surface (R3) is suppobedp zo /6ethe1fatedetermining step in the whole catalytic hydrolysis. The adsorption/desorption equilibrium is written by eqs. 1, 3, 4 and 7. The overall rate equation for catalytic hydrolysis can be

$$
k_{2}[p-\mathrm{NPP}]\left(1-\theta_{\text {total }}\right)=k_{-2} \theta_{p-\mathrm{NPP}}
$$

described as eq. 8 . The back reactions can be ignored at the

$$
\begin{aligned}
v & =k_{3} \theta_{\mathrm{H}_{2} \mathrm{O}} \theta_{p-\mathrm{NPP}}-k_{-3} \theta_{p-\mathrm{NP}} \theta_{\mathrm{Phos}} \\
& =\frac{k_{3} K_{1^{\prime}} K_{2}[p-\mathrm{NPP}]-k_{-3} K_{4} K_{5}[p-\mathrm{NP}][\mathrm{Phos}]}{\left(1+K_{1}^{\prime}+K_{2}[p-\mathrm{NPP}]+K_{4}[p-\mathrm{NP}]+K_{5}[\mathrm{Phos}]\right)^{2}}
\end{aligned}
$$

beginning of hydrolysis $([p-\mathrm{NP}] \simeq 0$, $[\mathrm{Phos}] \simeq 0)$ to obtain the initial reaction rate $\left(v_{0}\right)$ as described in eq. 9 which gives a

$$
v_{0}=\frac{k_{3} K_{1}^{\prime} K_{2}[p-\mathrm{NPP}]_{0}}{\left(1+K_{1}+K_{2}[p-\mathrm{NPP}]_{0}\right)^{2}}
$$

linear function between $[p-\mathrm{NPP}]_{0}$ and $\sqrt{\frac{[p-\mathrm{NPP}]_{0}}{v_{0}}}$ (eq. 10).

$$
\sqrt{k_{3} K_{1}{ }^{\prime} K_{2}} \sqrt{\frac{[p-\mathrm{NPP}]_{0}}{v_{0}}}=K_{2}[p-\mathrm{NPP}]_{0}+K_{1}{ }^{\prime}+1
$$

Hydrolysis of $p$-NPP was carried out with changing the initial concentration of $p$-NPP $\left([p-\mathrm{NPP}]_{0}: 5-25 \mathrm{mM}\right)$ in a HEPES buffer $(100 \mathrm{mM}, \mathrm{pH} 8.3,0.75 \mathrm{~mL})$ containing $\mathrm{Fe}^{\mathrm{III}}\left[\mathrm{Co}^{\mathrm{III}}(\mathrm{CN})_{6}\right](12.1 \mathrm{mg})$ at $60{ }^{\circ} \mathrm{C}$. The initial reaction rate, $v_{0}$, is directly proportional to $[p-\mathrm{NPP}]_{0}$ as predicted by eq. 6 however, no clear relationship was observed between

$\sqrt{\frac{[p-\mathrm{NPP}]_{0}}{v_{0}}}$ and $[p-\mathrm{NPP}]_{0}$ (Fig. 3), suggesting that surface adsorption of the substrates is the rate-determining step.

Robustness of $\mathrm{Co}^{\mathrm{III}}\left[\mathrm{Fe}^{\mathrm{III}}(\mathbf{C N})_{6}\right]$. The robustness of $\mathrm{Fe}^{\mathrm{III}}\left[\mathrm{Co}^{\mathrm{III}}(\mathrm{CN})_{6}\right]$ was examined by recycling tests for hydrolysis of $p$-NPP (Fig. 4a). The conversions and the initial reaction rates $\left(v_{0}\right)$ obtained for the reaction system using $\mathrm{Fe}^{\mathrm{III}}\left[\mathrm{Co}^{\mathrm{III}}(\mathrm{CN})_{6}\right]$ were $84 \%$ and $5.3 \times 10^{-3} \mathrm{~mol} \mathrm{~L}^{-1} \mathrm{~h}^{-1}$ at the $1^{\text {st }}$ run, $61 \%$ and $3.6 \times 10^{-3} \mathrm{~mol} \mathrm{~L}^{-1} \mathrm{~h}^{-1}$ at the $2^{\text {nd }}$ run, and $38 \%$ and $1.9 \times 10^{-3} \mathrm{~mol} \mathrm{~L}^{-1} \mathrm{~h}^{-1}$ at the $3^{\text {rd }}$ run, respectively. The slightly higher initial reaction rate at the $2^{\text {nd }}$ run compared with that at the $1^{\text {st }}$ run could result from increase of effective surface area by decreasing particles sizes during the reaction as evidenced by dynamic light scattering (DLS) measurements. The mean particles size of $\mathrm{Fe}^{\mathrm{III}}\left[\mathrm{Co}^{\mathrm{III}}(\mathrm{CN})_{6}\right]$ before reaction was $c a .390$ $\mathrm{nm}$, which is larger than that after the $1^{\text {st }}$ run $(235 \mathrm{~nm})$, with
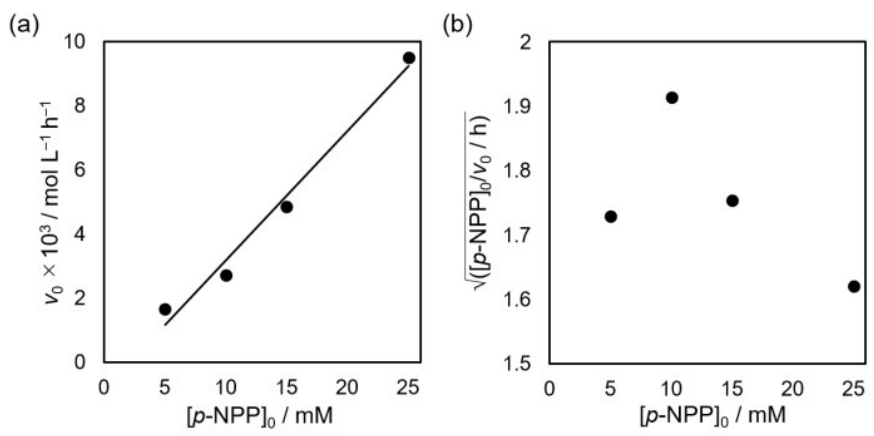

Fig. 3 (a) Relationship between initial rate for hydrolysis of $p$-NPP $\left(v_{0}\right)$ and initial concentrations of $p$-nitrophenyl phosphate $\left([p-N P P]_{0}\right)$. (b) Relationship between and

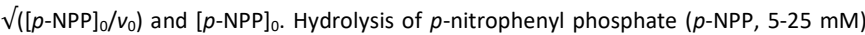
was carried out in a HEPES buffer $(100 \mathrm{mM}, \mathrm{pH} 8.3,0.75 \mathrm{~mL})$ containing Fe"'[ $\left[\mathrm{Co}{ }^{\text {III }}(\mathrm{CN})_{6}\right]$ (12.1 mg) at $60^{\circ} \mathrm{C}$ 

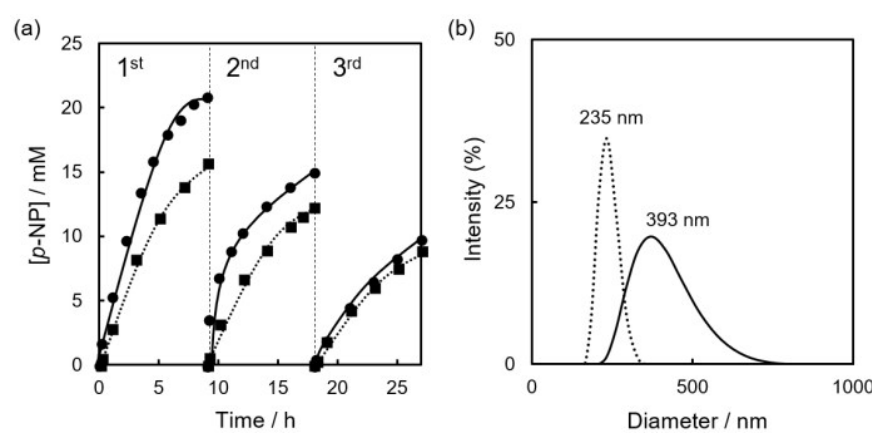

Fig. 4 (a) Time profiles of the formation of $p$-nitrophenol ( $p$-NP) by hydrolysis of $p$

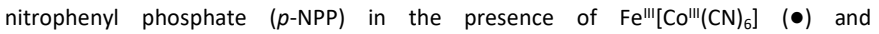
$\left[\mathrm{Fe}^{\prime \prime \prime}\left(\mathrm{H}_{2} \mathrm{O}\right)_{1.5}\right]_{4 / 3}\left[\mathrm{Fe}^{\prime \prime}(\mathrm{CN})_{6}\right](\mathbf{\square})$ in the repetitive experiments. Hydrolysis of $p$-nitrophenyl phosphate ( $p$-NPP, $25 \mathrm{mM}$ ) was performed in a HEPES buffer $(100 \mathrm{mM}, \mathrm{pH} 8.3,0.75$ $\mathrm{mL}$ ) containing catalysts $\left([\right.$ Substrate $\left.] /\left[\mathrm{Fe}^{\mathrm{III}}\right]=100\right)$. The complexes were recovered by centrifugation after each run and used for the reactions under the same conditions. (b) Particles size distribution of $\mathrm{Fe}^{\text {III' }}\left[\mathrm{Co}^{\text {III }}(\mathrm{CN})_{6}\right]$ dispersed in water before reactions (solid line) and after the first run (dotted line) obtained by dynamic light scattering (DLS)

wide size distribution (Fig. 4b). However, the conversion gradually decreased at the $2^{\text {nd }}$ and $3^{\text {rd }}$ runs. Metal oxide catalysts such as ceria and titania used for the catalytic hydrolysis of organophosphates in literature are expected to exhibit high durability under the reaction conditions. However, metal complex catalysts are prone to decomposition as reported in literature. ${ }^{43}$ The reaction systems using $\mathrm{Fe}^{\mathrm{III}}\left[\mathrm{Co}^{\mathrm{III}}(\mathrm{CN})_{6}\right]$ and $\left[\mathrm{Fe}^{\mathrm{III}}\left(\mathrm{H}_{2} \mathrm{O}\right)_{1.5}\right]_{4 / 3}\left[\mathrm{Fe}^{\mathrm{II}}(\mathrm{CN})_{6}\right]$ showed gradual decrease in conversions of $p$-NPP during three repetitive runs (Fig. 4a), implying that a part of cyano-bridged polynuclear metal complexes was also gradually degraded to less active forms during the reactions. ${ }^{43}$ IR spectrum of $\mathrm{Fe}^{\mathrm{III}}\left[\mathrm{Co}^{\mathrm{III}}(\mathrm{CN})_{6}\right]$ after the $1^{\text {st }}$ run showed the $v_{\mathrm{CN}}$ peak at $2169 \mathrm{~cm}^{-1}$ although the $v_{\mathrm{CN}}$ peak disappeared for the complex after the $3^{\text {rd }}$ run. Thus, partial decomposition of $\mathrm{Fe}^{\mathrm{III}}\left[\mathrm{Co}^{\mathrm{III}}(\mathrm{CN})_{6}\right]$ and $\left[\mathrm{Fe}^{\mathrm{III}}\left(\mathrm{H}_{2} \mathrm{O}\right)_{1.5}\right]_{4 / 3}\left[\mathrm{Fe}^{\mathrm{II}}(\mathrm{CN})_{6}\right]$ during reactions is a reason for the deceleration of catalytic hydrolysis.

Effects of temperature, $\mathrm{pH}$, reaction solvent and morphologies of $\left[\mathrm{Fe}^{\mathrm{III}}\left(\mathrm{H}_{2} \mathrm{O}\right)_{1.5}\right]_{4 / 3}\left[\mathrm{Fe}^{\mathrm{II}}(\mathrm{CN})_{6}\right]$ on catalysis. Catalysis of $\mathrm{Fe}^{\mathrm{III}}\left[\mathrm{Co}^{\mathrm{III}}(\mathrm{CN})_{6}\right]$ was examined at various temperatures between 40 and $70{ }^{\circ} \mathrm{C}$ (Fig. 5). The conversion of $p$-NPP in $10 \mathrm{~h}$ gradually increased with temperature reached $80 \%$ for $10 \mathrm{~h}$ at $70{ }^{\circ} \mathrm{C}$.

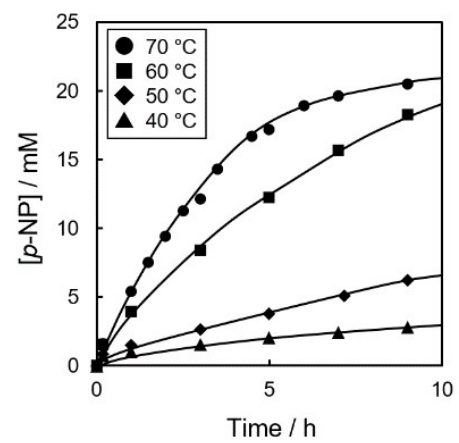

Fig. 5 Time profiles of $p$-nitrophenol ( $p$-NP) formation by hydrolysis of $p$-nitrophenyl phosphate ( $p$-NPP, $25 \mathrm{mM})$ in a HEPES buffer $(100 \mathrm{mM}, \mathrm{pH} 8.3,0.75 \mathrm{~mL})$ containing $\left.\mathrm{Fe}^{\mathrm{III}[\mathrm{Co}} \mathrm{Co}^{\mathrm{II}}(\mathrm{CN})_{6}\right]$ ([Substrate]/[Fe] $\left.=100\right)$ at temperatures of $40,50,60$ and $70^{\circ} \mathrm{C}$.
The effect of $\mathrm{pH}$ on catalytic hydrolysis of $p_{1}-\mathrm{NP}_{\mathrm{A}} \mathrm{P}_{\mathrm{ic}}$ in the

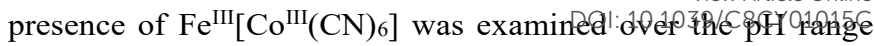
from 6 to 10 (Fig. 6). The conversion of $p$-NPP performed in the presence of $\mathrm{Fe}^{\mathrm{III}}\left[\mathrm{Co}^{\mathrm{III}}(\mathrm{CN})_{6}\right]$ exceeded $80 \%$ in $10 \mathrm{~h}$ at $\mathrm{pH}>$ 8 although the conversions significantly lowered to $\sim 20 \%$ under the conditions of $\mathrm{pH} 6$ to 7 , in which $v_{0}$ under basic conditions is more than double that of $\mathrm{pH} 7$.

The hydrolysis of $p$-NPP in a mixture of ethanol and HEPES buffer $(\mathrm{pH} 8.3, \mathrm{v} / \mathrm{v}=2 / 1)$ in the presence of $\mathrm{Fe}^{\mathrm{III}}\left[\mathrm{Co}^{\mathrm{III}}(\mathrm{CN})_{6}\right]$ was slightly slower than that in pure HEPES buffer although the initial rate was comparable to that of complete HEPES buffer system (Fig. 7a). On the other hand, the activity of $\left[\mathrm{Fe}^{\mathrm{III}}\left(\mathrm{H}_{2} \mathrm{O}\right)_{1.5}\right]_{4 / 3}\left[\mathrm{Fe}^{\mathrm{II}}(\mathrm{CN})_{6}\right]$ for catalytic hydrolysis in the mixed solvent was comparable to that in pure HEPES buffer (Fig. 7b), although $\left[\mathrm{Fe}^{\mathrm{III}}\left(\mathrm{H}_{2} \mathrm{O}\right)_{1.5}\right]_{4 / 3}\left[\mathrm{Fe}^{\mathrm{II}}(\mathrm{CN})_{6}\right]$ is less active than $\mathrm{Fe}^{\mathrm{III}}\left[\mathrm{Co}^{\mathrm{III}}(\mathrm{CN})_{6}\right]$ because of lower oxidation state of the $\mathrm{M}^{\mathrm{N}}$ ion. As described above, the rate determining step of the reaction is the adsorption of substrate on the catalyst surface. The lower activity of $\left[\mathrm{Fe}^{\mathrm{III}}\left(\mathrm{H}_{2} \mathrm{O}\right)_{1.5}\right]_{4 / 3}\left[\mathrm{Fe}^{\mathrm{II}}(\mathrm{CN})_{6}\right]$ in the mixed solvent indicates that ethanol adsorption disturbs substrate adsorption. The small effect of the reaction solvents on the catalytic activity of $\left[\mathrm{Fe}^{\mathrm{III}}\left(\mathrm{H}_{2} \mathrm{O}\right)_{1.5}\right]_{4 / 3}\left[\mathrm{Fe}^{\mathrm{II}}(\mathrm{CN})_{6}\right]$ suggests that no competitive adsorption of the substrate and ethanol occurs.

Effect of morphologies of $\left[\mathrm{Fe}^{\mathrm{III}}\left(\mathrm{H}_{2} \mathrm{O}\right)_{1.5}\right]_{4 / 3}\left[\mathrm{Fe}^{\mathrm{II}}(\mathrm{CN})_{6}\right]$ (Prussian blue) on catalytic activity was examined using Prussian blue nanoparticles in cubic shape (PB-cube) and in
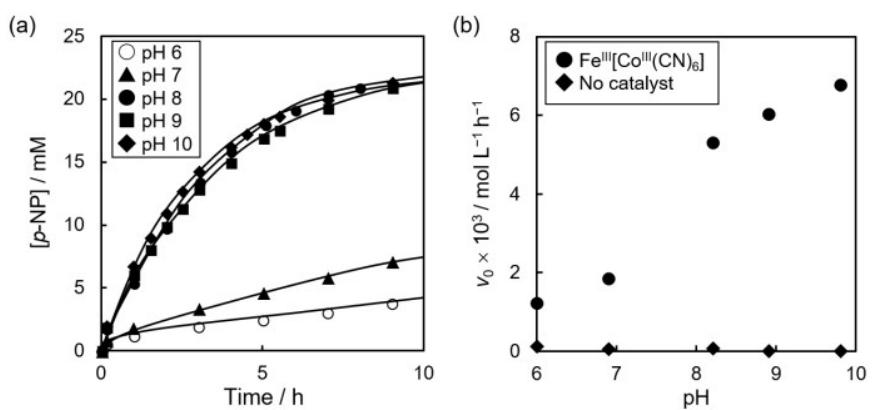

Fig. 6 (a) Time profiles of $p$-nitrophenol ( $p$-NP) formation by hydrolysis of $p$-nitropheny phosphate ( $p$-NPP, $25 \mathrm{mM}$ ) in a HEPES buffer $(100 \mathrm{mM}, \mathrm{pH} 6-10,0.75 \mathrm{~mL})$ containing $\mathrm{Fe}^{\prime \prime \prime \prime}\left[\mathrm{Co}{ }^{\prime \prime \prime}(\mathrm{CN})_{6}\right]([$ Substrate $] /[\mathrm{Fe}]=100)$ at $60^{\circ} \mathrm{C}$. (b) The plot of the initial reaction rate $\left(v_{0}\right)$ vs $\mathrm{pH}$.
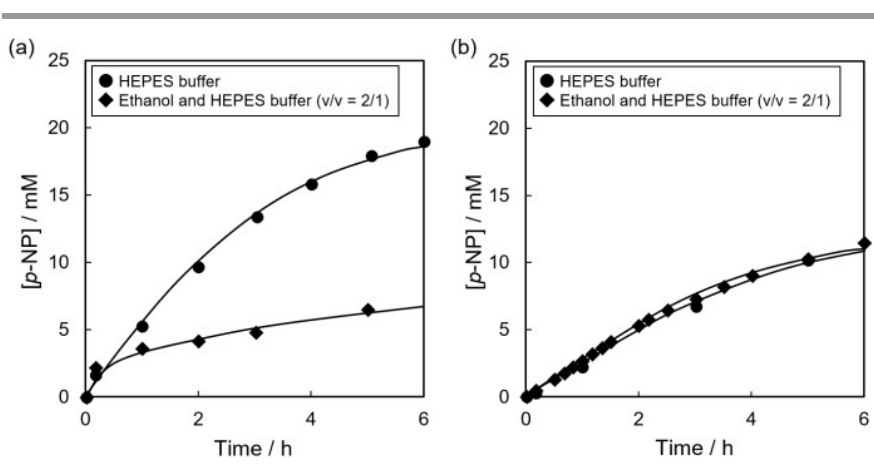

Fig. 7 Time profiles of the formation of $p$-nitrophenol ( $p$-NP) by hydrolysis of $p$ nitrophenyl phosphate ( $p$-NPP, $25 \mathrm{mM})$ in HEPES buffer $(0.75 \mathrm{~mL}, \mathrm{pH} 8.3$ ) or mixture of ethanol and HEPES buffer $(\mathrm{pH}$ 8.3) $(0.75 \mathrm{~mL}, \mathrm{v} / \mathrm{v}=2 / 1)$ in the presence of (a) $\mathrm{Fe}^{\mathrm{III}}\left[\mathrm{Co} \mathrm{Co}^{\prime \prime \prime}(\mathrm{CN})_{6}\right]$ and $(\mathrm{b})\left[\mathrm{Fe}^{\prime \prime \prime}\left(\mathrm{H}_{2} \mathrm{O}\right)_{1.5}\right]_{4 / 3}\left[\mathrm{Fe}\right.$ "' $\left.(\mathrm{CN})_{6}\right]$ at $60^{\circ} \mathrm{C}$. 
spherical shape (PB-sphere). ${ }^{77,78}$ The morphologies and size distributions of $\mathrm{PB}$ nanoparticles were confirmed by scanning electron microscope (SEM) measurements (Fig. 8a-c). The $v_{\mathrm{CN}}$ peaks in IR spectra and unit cell parameters determined by $\mathrm{XRD}$ of PB-cube and PB-sphere are comparable to those of Prussian blue prepared by a conventional method (PB, Table S2). The sharper XRD peaks observed for PB-cube and PBsphere evidenced their higher crystallinity than PB (Fig. S8). PB-cube and PB-sphere exhibited $\mathrm{N}_{2}$ adsorption-desorption isotherms of the type I form, suggesting the absence of mesoor macropores (Fig. S9 and Table S2). These results suggest that PB-cube and PB-sphere are highly crystalline. The highest $p$-NPP conversion of $79 \%(24 \mathrm{~h})$ with $v_{0}$ of $3.1 \times 10^{-3} \mathrm{~mol} \mathrm{~L}^{-1}$ $\mathrm{h}^{-1}$ was obtained for the reaction system using PB (Fig. 8d). Lower crystallinity of PB with many defect sites may contribute to the acceleration of the reaction. The defect sites of cyano-bridged polynuclear metal complexes are generated on $\mathrm{M}^{\mathrm{N}}$ ions, because $\mathrm{M}^{\mathrm{C}}$ ions in $\mathrm{M}^{\mathrm{C}}(\mathrm{CN})_{6}$ tend to maintain the coordinatively saturated structure without extra-ligand. The $\mathrm{M}^{\mathrm{N}}$ ions at defect sites are coordinated by water molecules as extraligand exchangeable with substrate molecules. The lower crystallinity of PB increases the average number of coordination of water molecules on $\mathrm{M}^{\mathrm{N}}$, resulting in high catalytic activity.

Evaluation of surface acidity by temperatureprogrammed desorption using pyridine. Basic conditions of reaction media accelerate hydrolysis of $p$-NPP as described above. However, acidic properties of catalytic surfaces are important for polarisation of phosphorus-oxygen bond of $p$ NPP, which succumbs to nucleophilic attack by $\mathrm{OH}^{-} .{ }^{40}$ Surface acidity of each complex was evaluated by heat of desorption of pyridine $(\Delta H \mathrm{~s})$ determined by the temperature-programmed desorption (TPD). ${ }^{80-83}$ The used amount of a sample and pretreatment temperature for the TPD measurements were

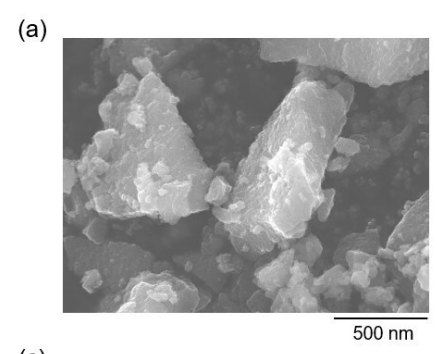

(c)

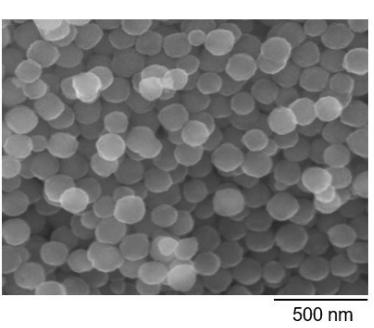

(b)

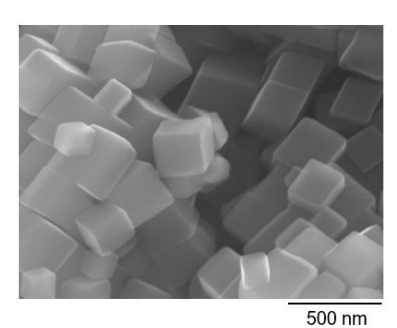

(d) 25

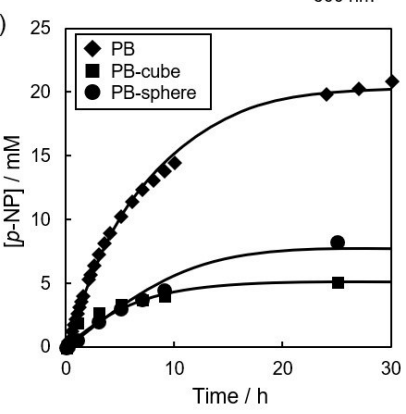

Fig. 8 SEM images of (a) PB, (b) PB-cube and (c) PB-sphere. (d) Time profiles of $p$ nitrophenol ( $p$-NP) formation by hydrolysis of $p$-nitrophenyl phosphate ( $p$-NPP, $25 \mathrm{mM}$ ) in a HEPES buffer (100 mM, pH 8.3, $0.75 \mathrm{~mL}$ ) containing PB in different morphologies ([Substrate $] /[\mathrm{Fe}]=100)$ at $60^{\circ} \mathrm{C}$. carefully chosen (Fig. S10 and S11), because the ratiogtef used

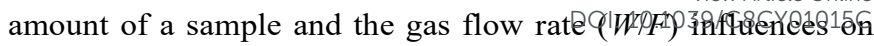
the temperature of pyridine-desorption maximum $\left(T_{\mathrm{M}}\right)$, and appropriate pretreatment temperature is important for complete removal of physisorbed water without decomposition of samples. $T_{\mathrm{M}}$ shifted by changing the ramp rate $(\beta)$ can be correlated with $\Delta H \mathrm{~s}$ by using eq. 11 for all cyano-bridged

$$
\ln \frac{T_{M}^{2}}{\beta}=\frac{\Delta H}{R T_{M}}+\ln \left[\frac{(1-\theta)^{2} V_{S} \Delta H}{F A R}\right]
$$

polynuclear metal complexes (Fig. S12), where $R, F, \beta, V_{\mathrm{s}}$ and $A$ stand for the gas constant, gas flow rate, coverage of catalyst surfaces, catalyst volume and pre-exponential factor, respectively. ${ }^{82} \Delta H \mathrm{~s}$ calculated from the slopes of the plots in Fig. 9a were $152,160,222,176,98 \mathrm{~kJ} \mathrm{~mol}^{-1}$ for $\left[\mathrm{Fe}^{\mathrm{III}}\left(\mathrm{H}_{2} \mathrm{O}\right)_{1.5}\right]_{4 / 3}\left[\mathrm{Fe}^{\mathrm{II}}(\mathrm{CN})_{6}\right]$, $\left[\mathrm{Mn}^{\mathrm{II}}\left(\mathrm{H}_{2} \mathrm{O}\right)_{2}\right]_{1.5}\left[\mathrm{Fe}^{\mathrm{III}}(\mathrm{CN})_{6}\right]$, $\left[\mathrm{Fe}^{\mathrm{II}}\left(\mathrm{H}_{2} \mathrm{O}\right)_{2}\right]_{1.5}\left[\mathrm{Co}^{\mathrm{III}}(\mathrm{CN})_{6}\right]$, respectively. Cyano-bridged polynuclear metal complexes with larger $\Delta H$ exhibited higher $v_{0}$ for hydrolysis of $p$-nitrophenyl phosphate ( $p$-NPP) (Fig. 9b and Table S3). The complexes with larger $\Delta H$ have stronger surface acidity, thus, the hydrolysis of organophosphates is accelerated by the cyano-bridged polynuclear metal complexes with strong surface acidity. The strong adsorption of organophosphate molecules to the surfaces of cyano-bridged polynuclear metal complexes accelerates the reaction, because the rate-determining step of hydrolysis is adsorption of organophosphates to the surface of cyano-bridged polynuclear metal complexes. However, $\left[\mathrm{Mn}^{\mathrm{II}}\left(\mathrm{H}_{2} \mathrm{O}\right)_{2}\right]_{1.5}\left[\mathrm{Fe}^{\mathrm{III}}(\mathrm{CN})_{6}\right]$ with the largest $\Delta H$ has the initial reaction rate slightly slower than expected. As mentioned in the previous section, strong surface acidity would decelerate desorption of adsorbed ethanol, thus, the strong surface acidity of $\left[\mathrm{Mn}^{\mathrm{II}}\left(\mathrm{H}_{2} \mathrm{O}\right)_{2}\right]_{1.5}\left[\mathrm{Fe}^{\mathrm{III}}(\mathrm{CN})_{6}\right]$ may decelerate the reaction by products inhibition.

\section{Conclusions}

Cyano-bridged polynuclear metal complexes prepared by systematic replacement of $\mathrm{M}^{\mathrm{N}}$ or $\mathrm{M}^{\mathrm{C}}$

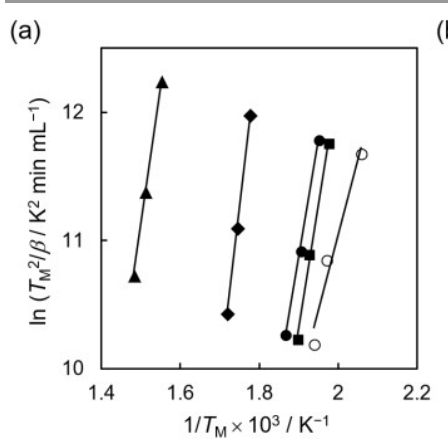

(b)

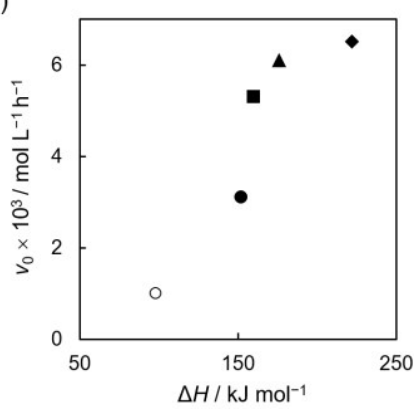

Fig. 9 (a) Plots of $\ln \left(T_{\mathrm{M}}^{2} / \beta\right)$ vs $1 / T_{\mathrm{M}}$, where $T_{\mathrm{M}}$ is the temperature of pyridine-desorption maximum; $\beta$ is the ramp rate, and (b) Relationship between the heat of pyridine desorption $(\Delta H)$ of $\left[\mathrm{Fe}^{\prime \prime \prime}\left(\mathrm{H}_{2} \mathrm{O}\right)_{1.5}\right]_{4 / 3}\left[\mathrm{Fe}^{\prime \prime \prime}(\mathrm{CN})_{6}\right] \quad(\bullet), \quad \mathrm{Fe}^{\prime \prime \prime \prime}\left[\mathrm{Co}^{\prime \prime \prime}(\mathrm{CN})_{6}\right] \quad(\mathbf{\square})$, $\left[\mathrm{Mn}^{\prime \prime}\left(\mathrm{H}_{2} \mathrm{O}\right)_{2}\right]_{1.5}\left[\mathrm{Fe}^{\prime \prime \prime}(\mathrm{CN})_{6}\right](\diamond), \mathrm{Ga}^{\prime \prime \prime}\left[\mathrm{Fe}^{\prime \prime \prime}(\mathrm{CN})_{6}\right](\boldsymbol{\Delta})$ and $\left[\mathrm{Fe}^{\prime \prime}\left(\mathrm{H}_{2} \mathrm{O}\right)_{2}\right]_{1.5}\left[\mathrm{Co}{ }^{\prime \prime \prime}(\mathrm{CN})_{6}\right](\mathrm{O})$ and the initial reaction rate $\left(v_{0}\right)$ for hydrolysis of $p$-nitrophenyl phosphate ( $p$-NPP, $25 \mathrm{mM}$ ) in a HEPES buffer $(100 \mathrm{mM}, \mathrm{pH} 8.3,0.75 \mathrm{~mL})$ containing each complex ([Substrate] $\left.] /\left[\mathrm{M}^{\mathrm{N}}\right]=100\right)$ at $60^{\circ} \mathrm{C}$. 
$\left(\left[\mathrm{M}^{\mathrm{N}}\left(\mathrm{H}_{2} \mathrm{O}\right)_{\mathrm{x}}\right]_{\mathrm{y}}\left[\mathrm{Fe}^{\mathrm{II} / \mathrm{III}}(\mathrm{CN})_{6}\right] ; \mathrm{M}^{\mathrm{N}}=\mathrm{Fe}^{\mathrm{III}}, \mathrm{Ga}^{\mathrm{III}}, \mathrm{Mn}^{\mathrm{II}}, \mathrm{Zn}^{\mathrm{II}}\right.$ or $\mathrm{Co}^{\mathrm{II}}$ : $\left[\mathrm{Fe}^{\mathrm{II} / \mathrm{III}}\left(\mathrm{H}_{2} \mathrm{O}\right)_{\mathrm{x}}\right]_{\mathrm{y}}\left[\mathrm{M}^{\mathrm{C}}(\mathrm{CN})_{6}\right] ; \mathrm{M}^{\mathrm{C}}=\mathrm{Fe}^{\mathrm{II}}, \mathrm{Pt}^{\mathrm{IV}}, \mathrm{Co}^{\mathrm{III}}, \mathrm{Ir}^{\mathrm{III}}$ or $\left.\mathrm{Ru}^{\mathrm{II}}\right)$ exhibited catalytic activity for hydrolysis of organophosphates. Among them, $\mathrm{Fe}^{\mathrm{III}}\left[\mathrm{Co}^{\mathrm{III}}(\mathrm{CN})_{6}\right]$ exhibited the highest catalytic activity for hydrolysis of $p$-nitrophenyl phosphate where the initial reaction rate was 1000 times higher than that obtained without catalysts. Significant improvement of catalytic activity was achieved by employing $\mathrm{M}^{\mathrm{C}}$ and/or $\mathrm{M}^{\mathrm{N}}$ ions in high oxidation states. Cyano-bridged polynuclear metal complexes with stronger surface acidity exhibited higher catalytic activity for hydrolysis of organophosphates. Thus, fine tuning of surface acidity of coordination polymers by systematic replacement of metal ions is a promising way to achieve highly active catalysts for hydrolysis of harmful organophosphates.

\section{Conflicts of interest}

There are no conflicts to declare.

\section{Acknowledgements}

We are thankful to Prof. Yoshihisa Kaneko from Osaka City University for scanning electron microscope measurements. This work was supported by Innovative Science and Technology Initiative for Security, ATLA, Japan. to Y.Y. (No. J161000157); Koyanagi Foundation and JSPS KAKENHI to Y.Y. (No. JP16H02268) and to H.T. (No. JP17H07020).

\section{Notes and references}

1 F. Worek, J. Jenner and H. Thiermann, Chemical Warfare Toxicology: Volume 2: Management of Poisoning, The Royal Society of Chemistry, Cambridge, UK, 2016.

2 B. Ballantyne and T. C. Marrs, Clinical and Experimental Toxicology of Organophosphates and Carbamates, Elsevier Ltd., Amsterdam, Netherlands, 1992.

3 G. Mercey, T. Verdelet, J. Renou, M. Kliachyna, R. Baati, F. Nachon, L. Jean and P. Y. Renard, Acc. Chem. Res., 2012, 45, 756766.

4 N. Sharma and R. Kakkar, Adv. Mater. Lett., 2013, 4, 508-521.

5 B. M. Smith, Chem. Soc. Rev., 2008, 37, 470-478.

6 F. Matsumura and C. R. Krishna Murti, Biodegradation of Pesticides, Plenum Press, New York, USA, 1982.

7 S. L. Giles, J. G. Lundin, R. B. Balow, P. E. Pehrsson and J. H. Wynne, Appl. Catal. A, 2017, 542, 306-310.

8 L. Y. Kuo, A. Bennett and Q. L. Miao, Inorg. Chem., 2017, 56, $10013-10020$.

9 M. Purg, M. Elias and S. C. L. Kamerlin, J. Am. Chem. Soc., 2017, 139, 17533-17546.

10 S. Y. Ryu, J. W. Chung and S. Y. Kwak, RSC $A d v$., 2017, 7 , 9964-9974.

11 F. M. Raushel, Nature, 2011, 469, 310-311.

12 M. Alcalde, M. Ferrer, F. J. Plou and A. Ballesteros, Trends in Biotechnology, 2006, 24, 281-287.

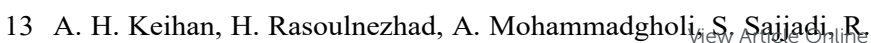

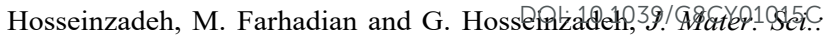
Mater. Electron., 2017, 28, 16718-16727.

14 T. Wang, J. N. Wang, Y. Yang, P. Su and Y. Yang, Ind. Eng. Chem. Res., 2017, 56, 9762-9769.

15 S. Kim, W. B. Ying, H. Jung, S. G. Ryu, B. Lee and K. J. Lee, Chem. -Asian. J., 2017, 12, 698-705.

16 Y. Y. Liu, A. J. Howarth, N. A. Vermeulen, S. Y. Moon, J. T. Hupp and O. K. Farha, Coord. Chem. Rev., 2017, 346, 101-111.

17 N. S. Bobbitt, M. L. Mendonca, A. J. Howarth, T. Islamoglu, J. T. Hupp, O. K. Farha and R. Q. Snurr, Chem. Soc. Rev., 2017, 46, 3357-3385.

18 S. S. Mondal and H. J. Holdt, Angew. Chem. Int. Ed., 2016, 55, $42-44$.

19 J. B. DeCoste and G. W. Peterson, Chem. Rev., 2014, 114, $5695-5727$.

20 F. J. Ma, S. X. Liu, C. Y. Sun, D. D. Liang, G. J. Ren, F. Wei, Y. G. Chen and Z. M. Su, J. Am. Chem. Soc., 2011, 133, 4178-4181.

21 T. Islamoglu, M. A. Ortuno, E. Proussaloglou, A. J. Howarth, N. A. Vermeulen, A. Atilgan, A. M. Asiri, C. J. Cramer and O. K. Farha, Angew. Chem. Int. Ed., 2018, 57, 1949-1953.

22 D. F. S. Gallis, J. A. Harvey, C. J. Pearce, M. G. Hall, J. B. DeCoste, M. K. Kinnan and J. A. Greathouse, J. Mater. Chem. A, 2018, 6, 3038-3045

23 D. L. McCarthy, J. Liu, D. B. Dwyer, J. L. Troiano, S. M. Boyer, J. B. DeCoste, W. E. Bernier and W. E. Jones, New J. Chem., 2017, 41, 8748-8753.

24. H. J. Park, J. K. Jang, S. Y. Kim, J. W. Ha, D. Moon, I. N. Kang, Y. S. Bae, S. Kim and D. H. Hwang, Inorg. Chem., 2017, 56, 12098-12101.

25 M. F. Xia, C. X. Zhuo, X. J. Ma, X. H. Zhang, H. M. Sun, Q. G. Zhai and Y. D. Zhang, Chem. Commun., 2017, 53, 11302-11305.

26 P. Asha, M. Sinha and S. Mandal, Rsc Adv, 2017, 7, 6691-6696.

27 M. C. de Koning, G. van Marco and T. Breijaert, Inorg. Chem., 2017, 56, 11804-11809.

28 D. T. Lee, J. J. Zhao, G. W. Peterson and G. N. Parsons, Chem. Mater., 2017, 29, 4894-4903.

29 T. Islamoglu, A. Atilgan, S. Y. Moon, G. W. Peterson, J. B. DeCoste, M. Hall, J. T. Hupp and O. K. Farha, Chem. Mater., 2017, 29, 2672-2675.

30 S. Y. Moon, E. Proussaloglou, G. W. Peterson, J. B. DeCoste, M. G. Hall, A. J. Howarth, J. T. Hupp and O. K. Farha, Chem. - Eur. J., 2016, 22, 14864-14868.

31 S. Y. Moon, A. J. Howarth, T. Wang, N. A. Vermeulen, J. T. Hupp and O. K. Farha, Chem. Commun., 2016, 52, 3438-3441.

32 P. Li, S. Y. Moon, M. A. Guelta, S. P. Harvey, J. T. Hupp and O. K. Farha, J. Am. Chem. Soc., 2016, 138, 8052-8055.

33 G. W. Peterson, S. Y. Moon, G. W. Wagner, M. G. Hall, J. B. DeCoste, J. T. Hupp and O. K. Farha, Inorg. Chem., 2015, 54, 9684-9686.

34 E. López-Maya, C. Montoro, L. M. Rodriguez-Albelo, S. D. A. Cervantes, A. A. Lozano-Perez, J. L. Cenis, E. Barea and J. A. R. Navarro, Angew. Chem. Int. Ed., 2015, 54, 6790-6794.

35 S. Y. Moon, Y. Y. Liu, J. T. Hupp and O. K. Farha, Angew. Chem. Int. Ed., 2015, 54, 6795-6799.

36 Y. Liu, S. Y. Moon, J. T. Hupp and O. K. Farha, ACS Nano, 2015, 9, 12358-12364. 
37 J. E. Mondloch, M. J. Katz, W. C. Isley, P. Ghosh, P. L. Liao, W. Bury, G. Wagner, M. G. Hall, J. B. DeCoste, G. W. Peterson, R. Q. Snurr, C. J. Cramer, J. T. Hupp and O. K. Farha, Nat. Mater., 2015, 14, 512-516.

38 P. Li, R. C. Klet, S. Y. Moon, T. C. Wang, P. Deria, A. W. Peters, B. M. Klahr, H. J. Park, S. S. Al-Juaid, J. T. Hupp and O. K. Farha, Chem. Commun., 2015, 51, 10925-10928.

39 S. Y. Moon, G. W. Wagner, J. E. Mondloch, G. W. Peterson, J. B. DeCoste, J. T. Hupp and O. K. Farha, Inorg. Chem., 2015, 54, 10829-10833.

40 M. J. Katz, R. C. Klet, S.-Y. Moon, J. E. Mondloch, J. T. Hupp, O. K. Farha, ACS Catal., 2015, 5, 4637-4642.

41 M. J. Katz, J. E. Mondloch, R. K. Totten, J. K. Park, S. T. Nguyen, O. K. Farha and J. T. Hupp, Angew. Chem. Int. Ed., 2014, 53, 497-501.

42 S. Wang, L. Bromberg, H. Schreuder-Gibson and T. A. Hatton, ACS Appl. Mater. Interfaces, 2013, 5, 1269-1278.

43 R. K. Totten, M. H. Weston, J. K. Park, O. K. Farha, J. T. Hupp and S. T. Nguyen, ACS Catal., 2013, 3, 1454-1459.

44 A. Roy, A. K. Srivastava, B. Singh, T. H. Mahato, D. Shah and A. K. Halve, Microporous Mesoporous Mater., 2012, 162, 207-212.

45 A. Roy, A. K. Srivastava, B. Singh, D. Shah, T. H. Mahato and A. Srivastava, Dalton Trans., 2012, 41, 12346-12348.

46 K. Kim, O. G. Tsay, D. A. Atwood and D. G. Churchill, Chem. Rev., 2011, 111, 5345-5403.

47 D. B. Dang, Y. Bai, C. He, J. Wang, C. Y. Duan and J. Y. Niu, Inorg. Chem., 2010, 49, 1280-1282.

48 R. Gil-San-Millan, E. López-Maya, M. Hall, N. M. Padial, G. W. Peterson, J. B. DeCoste, L. M. Rodriguez-Albelo, J. E. Oltra, E. Barea and J. A. R. Navarro, ACS Appl. Mater. Interfaces, 2017, 9 , 23967-23973.

49 M. J. Katz, S. Y. Moon, J. E. Mondloch, M. H. Beyzavi, C. J. Stephenson, J. T. Hupp and O. K. Farha, Chem. Sci., 2015, 6, 2286-2291.

50 S. Horike, M. Dincă, K. Tamaki and J. R. Long, J. Am. Chem. Soc., 2008, 130, 5854-5855.

51 J. C. Jiang and O. M. Yaghi, Chem. Rev., 2015, 115, 6966-6997.

52 B. Y. Li, K. Y. Leng, Y. M. Zhang, J. J. Dynes, J. Wang, Y. F. Hu, D. X. Ma, Z. Shi, L. K. Zhu, D. L. Zhang, Y. Y. Sun, M. Chrzanowski and S. Q. Ma, J. Am. Chem. Soc., 2015, 137, 4243-4248.

53 Z. G. Hu and D. Zhao, CrystEngComm, 2017, 19, 4066-4081.

54 Y. B. Huang, J. Liang, X. S. Wang and R. Cao, Chem. Soc. Rev., 2017, 46, 126-157.

55 P. F. Ji, X. Y. Feng, S. S. Veroneau, Y. Song and W. B. Lin, J. Am. Chem. Soc., 2017, 139, 15600-15603.

56 L. Jiao, Y. Wang, H. L. Jiang and Q. Xu, Adv. Mater, 2018, DOI: 10.1002/adma.201703663.

57 M. Witzel, B. Ziegler and D. Babel, Z. Anorg. Allg. Chem., 2000, 626, 471-477.

58 B. Bal, S. Ganguli and M. Bhattacharya, J. Phys. Chem., 1984, 88, 4575-4577.

59 B. Rodríguez-García, A. Reyes-Carmona, I. Jimenez-Morales, M. Blasco-Ahicart, S. Cavaliere, M. Dupont, D. Jones, J. Roziere, J. R. Galán-Mascarós and F. Jaouen, Sustainable Energy Fuels, 2018, 2, 589-597.

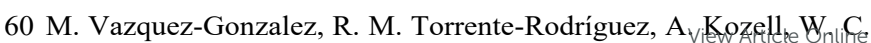

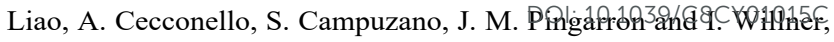
Nano Lett., 2017, 17, 4958-4963.

61 M. Aksoy, S. V. K. Nune and F. Karadas, Inorg. Chem., 2016, 55, 4301-4307.

62 K. Y. A. Lin, B. J. Chen and C. K. Chen, RSC $A d v$., 2016, 6, 92923-92933.

63 L. J. Han, P. Y. Tang, A. Reyes-Carmona, B. Rodríguez-García, M. Torrens, J. R. Morante, J. Arbiol and J. R. Galán-Mascarós, J. Am. Chem. Soc., 2016, 138, 16037-16045.

64 S. Goberna-Ferron, W. Y. Hernandez, B. Rodríguez-García and J. R. Galán-Mascarós, ACS Catal., 2014, 4, 1637-1641.

65 S. Pintado, S. Goberna-Ferrón, E. C. Escudero-Adan and J. R. Galán-Mascarós, J. Am. Chem. Soc., 2013, 135, 13270-13273.

66 Y. Aratani, T. Suenobu, K. Ohkubo, Y. Yamada and S. Fukuzumi, Chem. Commun., 2017, 53, 3473-3476.

67 Y. Yamada, K. Oyama, T. Suenobu and S. Fukuzumi, Chem. Commun., 2017, 53, 3418-3421.

68 Y. Isaka, K. Oyama, Y. Yamada, T. Suenobu and S. Fukuzumi, Catal. Sci. Technol., 2016, 6, 681-684.

69 Y. Yamada, K. Oyama, R. Gates and S. Fukuzumi, Angew. Chem. Int. Ed., 2015, 54, 5613-5617.

70 Y. Yamada, M. Yoneda and S. Fukuzumi, Inorg. Chem., 2014, 53, $1272-1274$

71 Y. Yamada, M. Yoneda and S. Fukuzumi, Chem. - Eur. J., 2013, 19, 11733-11741.

72 H. J. Buser, D. Schwarzenbach, W. Petter and A. Ludi, Inorg. Chem., 1977, 16, 2704-2710.

73 K. W. Chapman, P. J. Chupas and C. J. Kepert, J. Am. Chem. Soc., 2006, 128, 7009-7014.

74 S. S. Kaye and J. R. Long, Chem. Commun., 2007, 4486-4488.

75 M. S. Kandanapitiye, B. Valley, L. D. Yang, A. M. Fry, P. M. Woodward and S. P. D. Huang, Inorg. Chem., 2013, 52, 2790-2792.

76 Y. Aratani, K. Oyama, T. Suenobu, Y. Yamada and S. Fukuzumi, Inorg. Chem., 2016, 55, 5780-5786.

77 Y. D. Chiang, M. Hu, Y. Kamachi, S. Ishihara, K. Takai, Y. Tsujimoto, K. Ariga, K. C. W. Wu and Y. Yamauchi, Eur. J. Inorg. Chem., 2013, 12, 3141-3145.

78 X. P. Shen, S. K. Wu, Y. Liu, K. Wang, Z. Xu and W. Liu, J. Colloid Interface Sci., 2009, 329, 188-195.

79 K. Nakamoto, Infrared and Raman Spectra of Inorganic and Coordination Compounds, A Wiley-Interscience Publications, New York, USA, 4th edn, 1986.

80 M. Niwa and N. Katada, Chem. Rec., 2013, 13, 432-455.

81 M. Niwa, N. Katada, M. Sawa and Y. Murakami, J. Phys. Chem., 1995, 99, 8812-8816.

82 M. Nakano, T. Hironaka, S. Fujii and K. Sekizawa, Toyo Soda Kenkyu Hokoku, 1985, 29, 3-11.

83 V. R. Choudhary and V. S. Nayak, Mater. Chem. Phys., 1984, 11, 515-523. 